\title{
CHEMISTRY IN PROTOPLANETARY DISKS: A SENSITIVITY ANALYSIS
}

\author{
A. I. VASYUNIN \\ Max Planck Institute for Astronomy, Königstuhl 17, D-69117 Heidelberg, Germany; Ural State University, ul. Lenina 51, Yekatirinburg, \\ 620083, Russia; vasyunin@mpia.de \\ D. Semenov and Th. Henning \\ Max Planck Institute for Astronomy, Königstuhl 17, D-69117 Heidelberg, Germany; semenov@mpia.de, henning@mpia.de \\ V. WAKELAM \\ Université Bordeaux 1, CNRS, Laboratoire Astrophysique de Bordeaux, BP89 33270 Floirac, France; valentine.wakelam@obs.u-bordeaux1.fr
}

ERIC HERBST

Ohio State University, Department of Physics, 174 West 18th Avenue, Columbus, OH 43210-1106; herbst@mps.ohio-state.edu

AND

A. M. Sobolev

Ural State University, ul. Lenina 51, Yekatirinburg, 620083, Russia; andrej.sobolev@usu.ru

Received 2007 May 29; accepted 2007 September 20

\begin{abstract}
We study how uncertainties in the rate coefficients of chemical reactions in the RATE 06 database affect abundances and column densities of key molecules in protoplanetary disks. We randomly varied the gas-phase reaction rates within their uncertainty limits and calculated the time-dependent abundances and column densities using a gasgrain chemical model and a flaring steady state disk model. We find that key species can be separated into two distinct groups according to the sensitivity of their column densities to the rate uncertainties. The first group includes $\mathrm{CO}, \mathrm{C}^{+}$, $\mathrm{H}_{3}^{+}, \mathrm{H}_{2} \mathrm{O}, \mathrm{NH}_{3}, \mathrm{~N}_{2} \mathrm{H}^{+}$, and $\mathrm{HCNH}^{+}$. For these species the column densities are not very sensitive to the rate uncertainties, but the abundances in specific regions are. The second group includes $\mathrm{CS}, \mathrm{CO}_{2}, \mathrm{HCO}^{+}, \mathrm{H}_{2} \mathrm{CO}, \mathrm{C} 2 \mathrm{H}, \mathrm{CN}$, $\mathrm{HCN}, \mathrm{HNC}$, and other, more complex species, for which high abundances and abundance uncertainties coexist in the same disk region, leading to larger scatters in column densities. However, even for complex and heavy molecules, the dispersion in their column densities is not more than a factor of $\sim 4$. We perform a sensitivity analysis of the computed abundances to rate uncertainties and identify those reactions with the most problematic rate coefficients. We conclude that the rate coefficients of about a hundred chemical reactions need to be determined more accurately in order to greatly improve the reliability of modern astrochemical models. This improvement should be an ultimate goal of future laboratory studies and theoretical investigations.
\end{abstract}

Subject headings: accretion, accretion disks — astrochemistry — methods: statistical — molecular processes — planetary systems: protoplanetary disks

Online material: color figures

\section{INTRODUCTION}

More than 140 organic and inorganic molecular species consisting of up to 13 atoms have been identified in space so far, ${ }^{1}$ including such complex species as dimethyl ether $\left(\mathrm{CH}_{3} \mathrm{OCH}_{3}\right.$; Snyder et al. 1974) and acetamide $\left(\mathrm{CH}_{3} \mathrm{CONH}_{2}\right.$; Hollis et al. 2006). The rich variety of the observed molecules implies that many more relevant yet undiscovered species must be involved in the processes of their formation and destruction. Still, only a tiny fraction of these species have been firmly detected in several protoplanetary disks, including $\mathrm{CO}$ (and its isotopes), $\mathrm{CN}, \mathrm{HCN}, \mathrm{HNC}$, $\mathrm{H}_{2} \mathrm{CO}, \mathrm{C}_{2} \mathrm{H}, \mathrm{CS}, \mathrm{HCO}^{+}, \mathrm{H}^{13} \mathrm{CO}^{+}, \mathrm{DCO}^{+}$, and $\mathrm{N}_{2} \mathrm{H}^{+}$(Dutrey et al. 1997; Kastner et al. 1997; van Zadelhoff et al. 2001; Qi et al. 2003; Thi et al. 2004). Multimolecule, multitransition observations of emission lines with (sub)millimeter interferometers and single-dish antennas provide a wealth of information about planet-forming disks resembling the young Solar Nebula (Simon et al. 2000; Aikawa et al. 2003; Qi et al. 2005; Pety et al. 2006; Dutrey et al. 2007).

An ultimate goal of disk studies is the reconstruction of the evolutionary history and spatial abundance distribution of var-

\footnotetext{
${ }^{1}$ See http://astrochemistry.net.
}

ious species, which requires sophisticated chemical models (Willacy \& Langer 2000; Markwick et al. 2002; van Zadelhoff et al. 2003; Ilgner et al. 2004; Semenov et al. 2005; Aikawa \& Nomura 2006; Tscharnuter \& Gail 2007). The analysis and modeling of observational data acquired with limited spatial and spectral resolution is not complete without taking all possible uncertainties into account, such as instrumental errors, uncertainties in the distance to source, and orientation. Whereas these uncertainties can in general be reduced either by using better observational data provided by current interferometers (Plateau de Bure Interferometer, Submillimeter Array) equipped with a new generation of receivers or in the future with the advent of more powerful instruments like Atacama Large Millimeter Array, Expanded Very Large Array, and Herschel Space Observatory, there is an intrinsic source of ambiguity in all the chemical modelsour limited knowledge of the reaction rate coefficients.

Modern astrochemical databases include up to about 4500 gasphase reactions and $\sim 450$ species (Millar et al. 1997; Le Teuff et al. 2000; Smith et al. 2004; Woodall et al. 2007), but many of these reactions have poorly estimated rate coefficients with uncertainties of about a factor of 2 and larger. For example, radiative association reactions lead to the formation of new complex 
species out of smaller ones through the photon relaxation of an excited collisional molecular complex. Under low-density space conditions where three-body processes are unlikely, these reactions may have rate coefficients as high as $\sim 10^{-9} \mathrm{~cm}^{3} \mathrm{~s}^{-1}$ and as low as $\sim 10^{-17} \mathrm{~cm}^{3} \mathrm{~s}^{-1}$, depending on the density of vibrational states of the complex. The radiative association rates are usually difficult to measure in the laboratory as well as to predict theoretically, especially for bigger species (Bates 1951; Williams 1972; Herbst 1980).

In contrast, dissociative recombination of molecular ions almost always proceeds very rapidly, in particular at low temperature, with rate coefficients that can be accurately obtained (Florescu-Mitchell \& Mitchell 2006). However, the products and their branching channels of many dissociative recombination reactions are not known precisely, particularly at low temperatures (see, however, Semaniak et al. 2001; Ishii et al. 2006; Öjekull et al. 2006), which may spoil theoretical predictions (see, e.g., Millar et al. 1988; Geppert et al. 2005a).

Recently, Vasyunin et al. (2004, Paper I hereafter) investigated the influence of uncertainties in rate coefficients on molecular abundances in dense and diffuse clouds, using the gas-phase UMIST 95 database. They found that the abundance uncertainties of simple species are limited to within about an order of magnitude and increase substantially with the number of atoms in the molecule, although the uncertainties still do not exceed the observational errors for simple molecules. They proposed a sensitivity analysis to identify those reactions that introduce the largest errors in the computed concentrations. Wakelam et al. (2005, hereafter Paper II) studied uncertainties in the gas-phase chemistry of hot cores and demonstrated that at late times, $\gtrsim 10^{4} \mathrm{yr}$, modeled abundances of important molecules can suffer from large uncertainties due to poorly known rate coefficients. Wakelam et al. (2006a) focused on dense cloud chemistry and took uncertainties in the physical parameters into account, as well as comparing the osu.2003 and UMIST 99 chemistries. These authors came to the conclusion that grain-surface reactions should be considered in order to achieve better consistency with the observational data, even though there is a large degree of ambiguity in simple cloud models based on static physical and chemical structures. Finally, Wakelam et al. (2006b) expanded this study by using their uncertainty analysis to show that there is a bistability in the abundances of many species that are hypersensitive to the adopted value of the ratio of the cosmic-ray ionization rate of helium to that of hydrogen (see also Pineau des Forêts et al. 1992; Shalabiea \& Greenberg 1995; Boger \& Sternberg 2006). Furthermore, Izzard et al. (2007) recently studied the effect of proton-capture reaction rate uncertainties in the $\mathrm{NeNa}$ and $\mathrm{MgAl}$ chains on the abundances of the $\mathrm{Ne}, \mathrm{Na}, \mathrm{Mg}$, and $\mathrm{Al}$ isotopes produced in intermediatemass asymptotic giant branch stars.

Despite previous work in rate uncertainties, to the best of our knowledge there have been no attempts in the literature to study how these reaction uncertainties, which are unlikely to be eliminated in the near future, affect the results of astrochemical modeling in the wide range of physical conditions typical of protoplanetary disks. In this paper, we extend the previous analyses by Paper I and Paper II to the conditions of a low-mass protoplanetary disk surrounding a young T Tauri star. The aims of our study are several. First, we analyze how large the abundance and column density scatter for key species are due to uncertainties in rate coefficients. Second, we investigate how these uncertainties vary with disk location. Third, we isolate the reactions whose rate uncertainties contribute most to the abundance scatter and which are therefore worth studying in detail. Last, we predict how the overall consistency of theoretical models will be improved after these reaction rates are better constrained.

The organization of our paper is as follows. The adopted disk physical structure and time-dependent gas-grain chemical model are presented in $\S 2.1$. The Monte Carlo method used to introduce the uncertainties in the RATE 06 reaction rates is described in $\S 2.2$. In $\S 3$ we report computed distributions of the mean molecular abundances and column densities and their errors in the whole disk, and analyze the influence of the rate coefficient uncertainties on these quantities. A correlation method that allows determination of the role of various reactions in the abundance uncertainties as a function of time and disk location is outlined, and results for several key species are presented in $\S 4.1$. Particular attention is paid to the identification of the most uncertain reactions for the chemical evolution of key species in the entire disk. In $\S 4.2$ we predict to what extent the abundance scatter due to the rate uncertainties will be lowered when accurate rate values of several dozen of the most problematic reactions are available. The remainder of $\S 4$ is concerned with problems in different classes of reactions. Final conclusions are drawn in $\S 5$.

\section{DISK MODEL AND UNCERTAINTY APPROACH}

\subsection{Physical Structure and Chemical Model of the Disk}

In our simulations, we adopted the $1+1 \mathrm{D}$ steady state irradiated disk model with a vertical temperature gradient that represents the low-mass Class II protoplanetary disk surrounding the young T Tauri star DM Tau (D'Alessio et al. 1999). The disk has a radius of $800 \mathrm{AU}$, an accretion rate $\dot{M}=10^{-8} M_{\odot} \mathrm{yr}^{-1}$, a viscosity parameter $\alpha=0.01$, and a mass $M \simeq 0.07 M_{\odot}$ (Dutrey et al. 1997; Piétu et al. 2007). The thermal and density structure of the disk is shown in Figure 1. As a disk age we used a value of 5 Myr, which has been derived by Simon et al. (2000) based on evolutionary track modeling of the central star. In chemical simulations an outer disk region beyond the distance of $50 \mathrm{AU}$ from the central star is considered. This is the only routinely accessible disk region with existing (sub)millimeter interferometers.

We assumed that the disk is illuminated by UV radiation from the central star with an intensity $G=410 G_{0}$ at $r=100 \mathrm{AU}$ $\left[G(r) \propto r^{-0.5}\right]$ and by interstellar UV radiation with intensity $G_{0}$ in plane-parallel geometry (Draine 1978; van Dishoeck 1988; Bergin et al. 2003; Dutrey et al. 2007). The dust grains are assumed to be uniform $0.1 \mu \mathrm{m}$ spherical particles made of amorphous silicates with olivine stoichiometry (Semenov et al. 2003), with a dust-to-gas mass ratio of $1 \%$. The self-shielding and mutual shielding of $\mathrm{CO}$ and $\mathrm{H}_{2}$ against UV photodissociation is computed using the precalculated factors from Tables 10 and 11 in Lee et al. (1996).

Three other high-energy sources in the model that drive chemistry either through dissociation, ionization, or desorption are cosmic rays, decay of short-lived radionuclides, and stellar X-rays. The X-ray ionization rate in a given disk region is computed according to the results of Glassgold et al. (1997a, 1997b) with parameters for their high metal depletion case. In this model, the thermal $\sim 5 \mathrm{keV}$ X-ray photons are generated at $\sim 0.1$ AU above the central star, with a total X-ray luminosity of $\approx 10^{30} \mathrm{erg} \mathrm{cm}^{-2} \mathrm{~s}^{-1}$ (Glassgold et al. 2005). The cosmic-ray ionization rate is assumed to be $1.3 \times 10^{-17} \mathrm{~s}^{-1}$. The ionization rate caused by radionuclide decay (primarily ${ }^{26} \mathrm{Al}$ and ${ }^{60} \mathrm{Fe}$ ) is $6.5 \times 10^{-19} \mathrm{~s}^{-1}$ (Finocchi \& Gail 1997). We adding the X-ray and radionuclide ionization rates to those involving cosmic-ray particles (CRPs) and CRPinduced UV photons. 

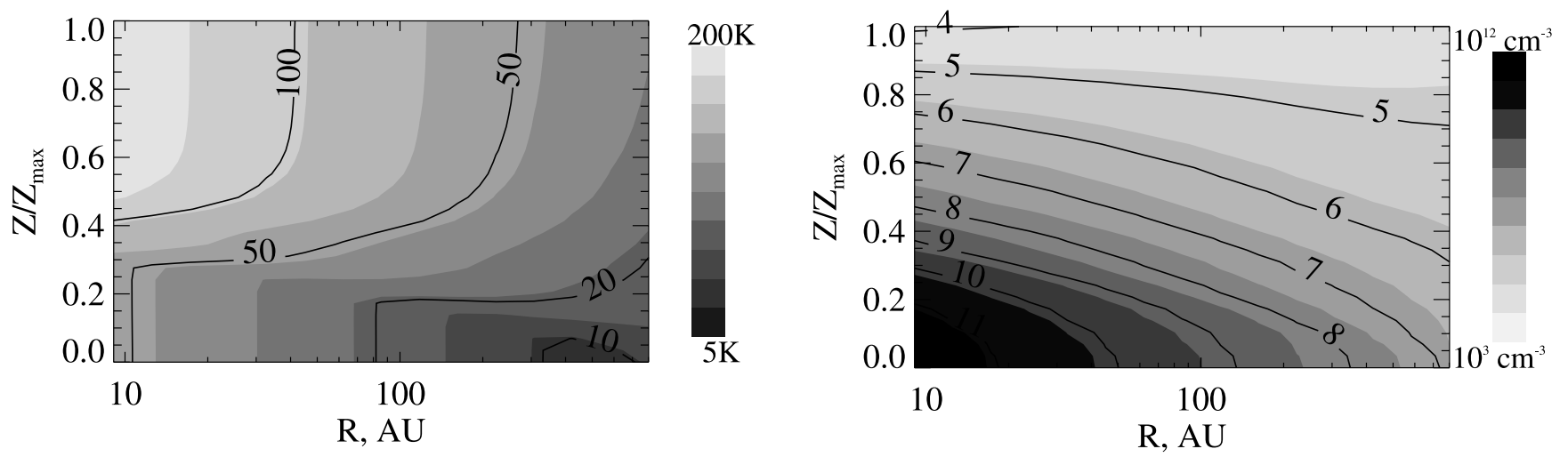

FIG. 1.- Temperature $(\mathrm{K})$ and density $\left(\mathrm{cm}^{-3}\right)$ structure of the adopted disk model. The total scale height of the disk $Z_{\max }$ increases with the radius such that at $r=100 \mathrm{AU}$ it has a value of $90 \mathrm{AU}$, whereas $Z_{\max } \approx 1300 \mathrm{AU}$ at $r=800 \mathrm{AU}$.

A modified static gas-grain chemical model has been utilized that includes gas-grain interactions without surface reactions or turbulent mixing (Semenov et al. 2005). We allow only surface formation of molecular hydrogen using the approach of Hollenbach $\&$ McKee (1979). This modification significantly decreases the numerical demands of our chemical code and makes it computationally tractable while allowing us to include all necessary physical processes. The gas-phase reaction rates and their uncertainties are taken from the recent RATE 06 database, in which the effects of dipole-enhanced ion-neutral rates are included (Woodall et al. 2007). To calculate photoreaction rates through the disk, we adopt precomputed fits of van Dishoeck (1988) instead of integrating the wavelength-dependent cross sections over the local UV spectrum (see van Zadelhoff et al. 2003).

Gas-grain interactions include the accretion of neutral molecules onto dust surfaces with a sticking efficiency of $100 \%$, dissociative recombination of ions on charged dust grains, and grain recharging, as well as UV-induced, CRP-induced, and thermal desorption of surface species. Desorption energies $E_{\mathrm{des}}$ are mostly taken from the recent osu. $2007^{2}$ database (Garrod \& Herbst 2006) or roughly estimated by analogy for about 20 molecules.

Overall, our network consists of positively charged and negatively charged as well as neutral dust grains, 420 gas-phase and 157 surface species made of 13 elements, and 5773 reactions. Among these 5773 reactions are 4517 gas-phase reactions, two charge exchange reactions for dust grains, 940 dissociative recombination reactions of ions on charged and neutral grains, and 314 accretion/desorption processes.

As initial abundances, we have adopted the so-called low-metal set of Lee et al. (1998) as listed in Table 1 and assumed that all hydrogen is mostly locked in its molecular form. In these initial abundances, the standard solar elemental composition is depleted in heavy elements by, e.g., 200 times for $\mathrm{S}$ up to more than $10^{4}$ for $\mathrm{Fe}, \mathrm{Cl}, \mathrm{P}$, and $\mathrm{F}$. The mostly atomic initial abundances are chosen instead of those from a molecular cloud model because this choice allows the abundance uncertainties to accumulate in a long sequence of chemical reactions with imprecise rates, starting from basic processes that reach steady state at early times during the disk evolution. Willacy et al. (1998) have shown that most of the molecular abundances are hardly affected by the choice of input abundances. Note also that the resulting abundance uncertainties can be sensitive to the initial elemental concentrations (Wakelam et al. 2006b). However, we do not allow the elemental concentrations to vary in order to keep the computations in reasonable limits.

\footnotetext{
${ }^{2}$ See https://www.physics.ohio-state.edu/ eric/research.html.
}

\subsection{Method to Model Rate Uncertainties}

Each reaction rate coefficient in the RATE 06 database is given in the standard Arrhenius form and thus relies on three parameters: $\alpha$ (the absolute value at room temperature), $\beta$ (the index for the power-law dependence of the rate on temperature), and $\gamma$ (the activation energy barrier in $\mathrm{K}$ ). A specific expression for the reaction rate coefficient depends on the type of chemical process: bimolecular reaction, direct cosmic-ray ionization reaction, photodissociation, etc. Note that in all cases the rate coefficient scales linearly with the parameter $\alpha$.

In addition to these three parameters, the RATE 06 reaction rates are characterized by an accuracy estimate (A, B, C, and D), where the uncertainties are smaller than $25 \%, 50 \%$, within a factor of 2, and within an order of magnitude. Almost all reactions with measured rates belong to the first group ( $\sim 1400$ reactions), while those reactions with rates that were "guessed" or derived by analogy are assigned to the third group ( $\sim 2800$ reactions), which contains most of the ion-neutral reaction rates. The second group includes about 300 mostly neutral-neutral and photodissociation reactions, whereas the fourth group consists of only four photodissociation reactions (Woodall et al. 2007).

In accord with the latest osu. 2007 network, we adopted a rate uncertainty of one order of magnitude for radiative association reactions. Such a large uncertainty factor is justified by the difficulty in calculating or measuring these rate coefficients (e.g., Herbst 1985). The uncertainty factors vary widely for the photoreaction

TABLE 1

INITIAL AbUNDANCES

\begin{tabular}{|c|c|}
\hline Species & $n(\mathrm{X}) / n(\mathrm{H})$ \\
\hline $\mathrm{He}$ & $9.75(-2)^{\mathrm{a}}$ \\
\hline $\mathrm{H}_{2}$ & $4.99(-1)$ \\
\hline  & $2.00(-3)$ \\
\hline $\mathrm{C} \ldots \ldots \ldots \ldots \ldots \ldots \ldots$ & $7.86(-5)$ \\
\hline $\mathrm{N}$ & $2.47(-5)$ \\
\hline 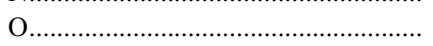 & $1.80(-4)$ \\
\hline $\mathrm{S}$ & $9.14(-8)$ \\
\hline 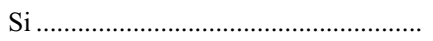 & $9.74(-9)$ \\
\hline $\mathrm{Na} \ldots \ldots \ldots \ldots$ & $2.25(-9)$ \\
\hline Mg & $1.09(-8)$ \\
\hline $\mathrm{Fe}$ & $2.74(-9)$ \\
\hline 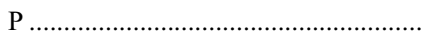 & $2.16(-10)$ \\
\hline $\mathrm{Cl}$ & $1.00(-9)$ \\
\hline $\mathrm{F}$ & $1.00(-10)$ \\
\hline
\end{tabular}


rates since the corresponding frequency-dependent cross sections have limited accuracy and have been obtained only for a fraction of molecules in the UMIST database (van Dishoeck 1988; van Dishoeck et al. 2006).

Our method for model abundance uncertainties is based on the computation of a large set of chemical models using identical physical and initial conditions and the same chemical network, but with randomly varied rate coefficients within their uncertainty limits. We utilized the same method to introduce uncertainties in the rate coefficient values as described in Dobrijevic \& Parisot (1998), Dobrijevic et al. (2003), and Paper II. The rate coefficient for each gas-phase reaction $i$ is randomly chosen over a lognormal distribution with median $\alpha_{i}$ and dispersion $F_{i}$. Consequently, the rate coefficient of the $i$ th reaction spans an interval between $\alpha_{i} / F_{i}$ and $\alpha_{i} F_{i}$ with a probability of $68 \%(1 \sigma)$. We generated a sequence of these lognormally distributed rates for all gas-phase reactions in our chemical network using the following expression:

$$
\alpha_{i}^{l}=\alpha_{i}\left(F_{i}\right)^{\epsilon},
$$

where $\alpha_{i}^{l}$ is the $l$ th realization of the $i$ th reaction rate; $\alpha_{i}$ is the standard RATE 06 rate value for the $i$ th reaction; $F_{i}$ is the dispersion, or the uncertainty factor, for this rate; and $\epsilon$ is randomly distributed from -1 to 1 with a uniform distribution law.

Using this approach, we allowed the rates of only 4517 gasphase reactions to vary in our network. The rates of all other processes (gas-grain interactions, dissociative recombination of molecular ions on grains, and recharging of dust grains) were kept constant. We did not account for possible variations among the parameters of the disk physical model. Such an idealization allowed us to focus solely on an investigation of how uncertainties in gas-phase reactions affect the results of the disk chemical modeling.

Using 8000 realizations of the RATE 06 network, we simulated 5 Myr of chemical evolution in the outer, $r \geq 50 \mathrm{AU}$, disk of DM Tau. The disk grid consists of five radial $(50,100,200$, 380 , and $760 \mathrm{AU}$ ) and 10 equidistant vertical points (with step sizes of 3.2, 7.6, 18, 41.7, and $96 \mathrm{AU}$ for the considered radii). Such a huge number of utilized chemical models with randomly varied rates leads to low values of statistical noise in the distributions of the abundance scatters at any particular disk location, assuring the correctness of the sensitivity analysis. Moreover, a large number of varied reaction rates allows an analysis of all reactions in the chemical network, in contrast to models with limited chemistry included in previous works (e.g., Paper II). Still, even with the modest $5 \times 10$ spatial resolution of the adopted disk model and without surface reactions, the overall computational time needed to calculate the chemistry with these 8000 networks was about 3 days on a four CPU PC machine $(3.0 \mathrm{GHz}$ Xeon, 8 Gbyte RAM).

\section{RESULTS}

\subsection{Abundance Distribution Profiles}

Before we perform a detailed analysis of the influence of the rate uncertainties on the modeled molecular abundances, we investigate the abundance distribution profiles. Because we utilized a lognormal distribution for the rate coefficients, one might expect that for each molecule the abundance distribution should show a normal (Gaussian) profile in logarithmic scale. If this hypothesis is correct, one can use the dispersion - a measure of the scatter of randomly varied values that have a Gaussian distribution - as a convenient quantity characterizing the abundance and column density uncertainties.
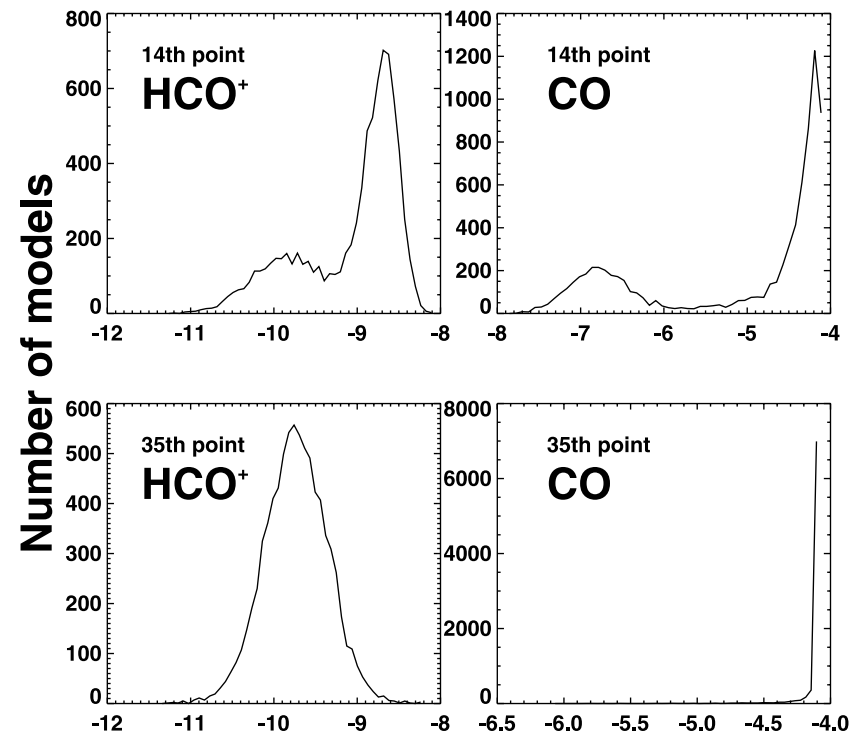

\section{$\log n(X) / n\left(H_{2}\right)$}

FIG. 2.-Histograms of the abundance distributions show the number of chemical models in which a given molecular abundance is reached at $5 \mathrm{Myr}$. Top: Non-Gaussian, double-peak distributions are shown for $\mathrm{HCO}^{+}$(left) and $\mathrm{CO}$ (right) in the inner disk region $(r \approx 97.5 \mathrm{AU}, z \approx 30 \mathrm{AU})$. Bottom: Nearly Gaussian distributions are shown for the same species but located farther out from the central $\operatorname{star}(r \approx 382 \mathrm{AU}, z \approx 205 \mathrm{AU})$. The point numbers in the panels refer to the disk cells for which these calculations were performed.

As shown in Paper II, this assumption may not be fulfilled for certain species and certain time steps. For example, when molecular abundances of a particular species show a steep decline or increase with time then at that moment the corresponding histogram of the abundance distribution can have several peaks and thus be far from a Gaussian shape because of sampling effects. Bimodal distributions can also be obtained if the system is highly sensitive to a small variation of specific parameter. Wakelam et al. (2006b) showed that dense cloud chemistry is hypersensitive, and even bistable, to the ratio between $\mathrm{He}$ and $\mathrm{H}_{2}$ cosmic-ray ionization rates in particular conditions. In this case our uncertainty method would tend to overestimate the dispersion of the modeled abundances. The disk chemistry is very rich in the sense that it proceeds vastly differently in various disk regions and at different times, so it is natural to expect that the abundance distributions of some species can sometime deviate from a normal distribution.

We carefully studied this problem and found that such a situation happens rarely at the late times, $\gtrsim 1 \mathrm{Myr}$, for which our analysis will be performed. In Figure 2 we show several representative histograms of the abundance distributions at $5 \mathrm{Myr}$, using $\mathrm{HCO}^{+}$and $\mathrm{CO}$ as examples. For an inner disk region, at $r \approx 97.5 \mathrm{AU}, z \approx 30 \mathrm{AU}$, a strong deviation from the Gaussian shape is clearly visible, with two distinct peaks and a gap in between (Fig. 2, top). Note that this is one of the few exceptional cases that is discussed in detail below. Everywhere else through the disk the $\mathrm{HCO}^{+}$and $\mathrm{CO}$ abundances have nearly Gaussianlike profiles (Fig. 2, bottom). This condition holds for other key species as well, implying that the averaged abundances in the disk should not differ from the values computed with the standard RATE 06 network. Moreover, in Paper I we found that the Gaussian shape of the abundance distribution is also preserved for the uniform distribution of the rate uncertainties in linear scale. 
In the rest of this paper, we will refer to one standard deviation $(1 \sigma)$ in the modeled abundance distribution as the abundance uncertainty. In our notation "abundance scatter" represents twice the abundance uncertainty in logarithmic scale $(2 \log \sigma)$. It is the abundance uncertainties that have to be taken into account when interpreting the observational data with a chemical model.

\subsection{Distribution of the Mean Abundances in the Disk}

Although the evolution of all species was calculated in 8000 realizations of the chemical model, in what follows we focus on several key molecules that are used as tracers of disk physical structure and chemical composition. Our list of important species includes molecules that are widely used for kinematic studies and determination of the density and temperature $\left(\mathrm{CO}, \mathrm{CS}, \mathrm{H}_{2} \mathrm{CO}\right.$, $\mathrm{NH}_{3}$, and $\mathrm{HCN}$ ) as well as for probing such major parameters as ionization degree $\left(\mathrm{HCO}^{+}\right)$, radiation fields $(\mathrm{CN}, \mathrm{HCN})$, and the yet-to-be detected $\mathrm{C}^{+}$as a dominant ion in the disk surface (Dutrey et al. 1997, 2007; Kastner et al. 1997; Aikawa et al. 2003).

We use time-dependent abundances to analyze the chemical evolution of these species in detail. The analysis is based on the calculation of the relative importance of each reaction in the chemical network for the abundance change of a molecule under investigation at a given time (Wiebe et al. 2003). This allows us to weight all reactions in the model and isolate a few of the most important pathways for chemical evolution of one or several species under specific physical conditions. In the case of protoplanetary disks, such an analysis has to be performed for several tens of representative cells.

In Figure 3 we show the mean abundances of these molecules in the disk at $5 \mathrm{Myr}$, which are obtained by averaging the results of the chemical network. The vertical extension of the disk is scaled by one hydrostatic scale height, $H(r)=\sqrt{2} C_{s} / \Omega$, where $C_{s}$ is the midplane sound speed and $\Omega$ is the Keplerian frequency (Dartois et al. 2003). The unity pressure scale height is about 25 and $320 \mathrm{AU}$ at distances of 100 and $800 \mathrm{AU}$, respectively.

The "sandwich-like" chemical structure of the disk is clearly seen in Figure 3. In the "dark," cold, and dense midplane at $\lesssim 0.5-1$ pressure scale height, the molecules freeze out onto dust grains within $100-100,000 \mathrm{yr}$ and low steady state fractional abundances of $\sim 10^{-14}$ to $10^{-10}$ are reached. The abundances of the gas-phase species in the midplane at late times are sustained by the CRP-induced desorption of the mantle materials. The midplane abundance of $\mathrm{CO}, \sim 10^{-8}$, is higher because this molecule is very abundant $\left(\approx 10^{-4}\right)$ in the gas phase initially, and since $\mathrm{CO}$ ice is rather volatile because of its low $\mathrm{CO}$ desorption barrier of $\sim 930 \mathrm{~K}$ (Bisschop et al. 2006). Overall, the gas-phase abundances in the midplane tend to slightly increase with radius as the disk surface density and thus the density in midplane decrease and gas-grain interactions become less intense, which slows down the freezeout of gas-phase molecules onto dust grain surfaces.

The surface layer of the disk $[Z / H(r) \geq 2]$ is deficient in molecules due to strong irradiation by high-energy stellar and interstellar photons. Even $\mathrm{CO}$, which is self-shielded and mutually shielded by $\mathrm{H}_{2}$ against UV photodissociation, cannot survive. Among the considered species, only $\mathrm{C}^{+}$reaches its maximal concentration and becomes the most abundant ion in the surface layer, with an abundance of $\sim 10^{-4}$. The thickness of this highly ionized layer $(\sim 1$ pressure scale height down from the disk surface) stays nearly constant with radius for all key species except highly reactive radicals $\mathrm{CS}$ and $\mathrm{CN}$, whose abundances are already high $\left(\sim 10^{-9}\right)$ at scale pressure heights as high as $Z / H(r) \approx 2$ at all radii (Fig. 3).

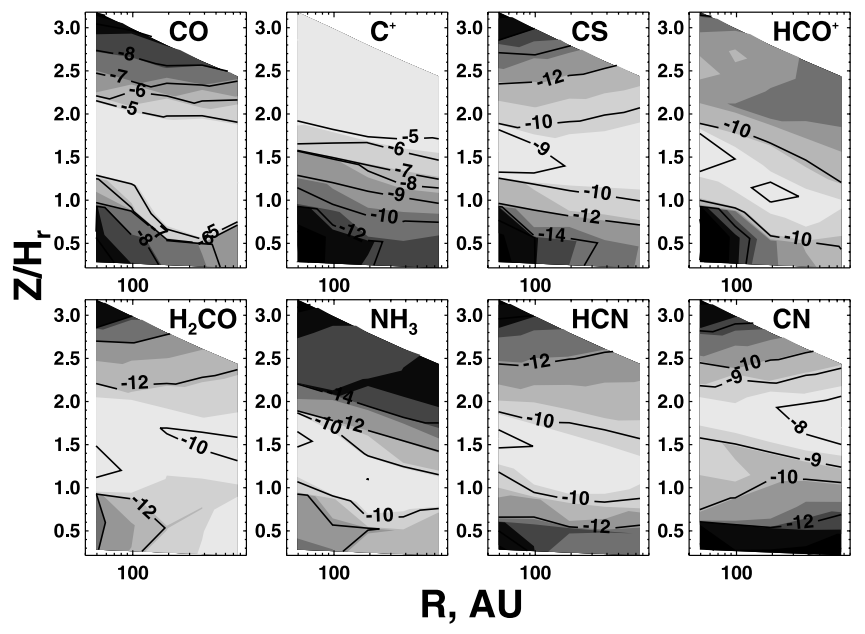

FIG. 3.-Distribution of mean molecular abundances relative to the total amount of hydrogen nuclei for several observationally important species in the disk at 5 Myr. The distributions are obtained by averaging the 8000 model results calculated with randomly varied reaction rates. Results are shown for $\mathrm{CO}, \mathrm{C}^{+}, \mathrm{CS}$, $\mathrm{HCO}^{+}$(top, from left to right) and $\mathrm{H}_{2} \mathrm{CO}, \mathrm{NH}_{3}, \mathrm{HCN}$, and $\mathrm{CN}$ (bottom) at $r \geq 50 \mathrm{AU}$. The disk vertical axis is given in units of the pressure scale height. [See the electronic edition of the Journal for a color version of this figure.]

In contrast, polyatomic species tend to concentrate in the warm, chemically rich intermediate layer $(\$ 1-2$ pressure scale heights, depending on the radius). The model-averaged abundances for the considered species, $\sim 10^{-10}$ to $10^{-4}$, are close to the values computed with the standard RATE 06 network and those found in previous work (Aikawa \& Herbst 1999; Willacy \& Langer 2000; Aikawa et al. 2002; Willacy et al. 2006; Dutrey et al. 2007).

Now let us consider the chemical processes that form and destroy the considered species in the intermediate layer. In contrast to dense cloud models, for many species in this disk zone steady state is reached by $\sim 10^{3}-10^{5}$ yr. This is due to the higher densities encountered in the warm layer $\left(\geq 10^{6}-10^{8} \mathrm{~cm}^{-3}\right)$ than in the cloud cores $\left(\sim 10^{4}-10^{6} \mathrm{~cm}^{-3}\right)$ and a non-negligible flux of the UV radiation. Impinging interstellar UV photons allow many surface species to desorb back into the gas phase (Hasegawa \& Herbst 1993; Garrod \& Herbst 2006), thus reducing their surface abundances and overall importance of gas-grain interactions for disk chemical evolution at late times, $t \gtrsim 10,000 \mathrm{yr}$.

The evolution of $\mathrm{CO}$ and $\mathrm{HCO}^{+}$in the intermediate layer is governed by a small set of reactions at all radii. The $\mathrm{CO}$ molecules are formed via reactions of atomic oxygen with $\mathrm{CH}, \mathrm{CH}_{2}$, and $\mathrm{C}_{2}$ at $t \lesssim 1000 \mathrm{yr}$, and partly through the dissociative recombination of $\mathrm{HCO}^{+}$afterward (at this stage steady state has been reached and there is a loop between $\mathrm{CO}$ and its protonated form). The light hydrocarbons that are precursors of $\mathrm{CO}$ are themselves rapidly created within a few years via the radiative association of $\mathrm{C}$ with $\mathrm{H}$ and $\mathrm{H}_{2}$, and $\mathrm{CH}$ with $\mathrm{H}_{2}$. The $\mathrm{CO}$ is mainly removed from the gas phase by accretion onto the dust grain surfaces in outer, cold regions and by reactive collisions with helium ions in the inner, X-ray ionized part of the intermediate layer. In the upper part of this layer, $\mathrm{CO}$ is also photodissociated. The ion $\mathrm{HCO}^{+}$initially forms via reactions of $\mathrm{O}$ with $\mathrm{CH}$ and ionized light hydrocarbons, $\mathrm{CH}_{n}^{+}(n=1-3)$, and the ion-neutral reaction between $\mathrm{CO}$ and $\mathrm{CH}_{5}^{+}$(also producing methane). At a later time of $\gtrsim 1000 \mathrm{yr}$, $\mathrm{HCO}^{+}$becomes the most abundant ion in the intermediate layer and the dominant molecular ion in the disk, with a fractional abundance of $\sim 10^{-9}$. At this steady state stage, $\mathrm{HCO}^{+}$is mainly produced via reactive collisions between $\mathrm{CO}$ and $\mathrm{H}_{3}^{+}$and destroyed by dissociative recombination with electrons and negatively charged grains. 
The chemically related $\mathrm{H}_{2} \mathrm{CO}$ molecule is produced through reaction of $\mathrm{CH}_{3}$ with oxygen atoms. At a later time of $\sim 10^{5} \mathrm{yr}$ $\mathrm{H}_{2} \mathrm{CO}$ is involved in a simple formation-destruction cycle. It starts with protonation of formaldehyde by $\mathrm{HCO}^{+}, \mathrm{H}_{3} \mathrm{O}^{+}$, and $\mathrm{H}_{3}^{+}$, which is followed by dissociative recombination into $\mathrm{H}_{2} \mathrm{CO}$ (33\%), $\mathrm{CO}(33 \%)$, or $\mathrm{HCO}(33 \%)$. Apart from photodissociation and freeze out, another direct destruction channel for $\mathrm{H}_{2} \mathrm{CO}$ in the upper, more ionized part of the intermediate layer is the ionneutral reaction with $\mathrm{C}^{+}$, which produces either $\mathrm{CO}$ and $\mathrm{CH}_{2}^{+}$, $\mathrm{HCO}^{+}$and $\mathrm{CH}$, or $\mathrm{H}_{2} \mathrm{CO}^{+}$and $\mathrm{C}$. The chemical evolution of formaldehyde typically reaches steady state at about $10^{5} \mathrm{yr}$ with a gas-phase abundance of $\lesssim 3 \times 10^{-10}$. Note that in the same disk chemical model as discussed above but with a set of surface reactions taken from Garrod \& Herbst (2006) the overall (gasphase and surface) abundance of formaldehyde is decreased by a factor of several as it is converted to methanol by hydrogenation on grain surfaces.

The photostable radical $\mathrm{CN}$ is abundant $\left(10^{-8}\right)$ at an upper, less molecularly rich disk layer $[Z / H(r) \approx 1.8$; Fig. 3]. The initial formation channel for the cyanogen radical is the radiative association between $\mathrm{C}$ and $\mathrm{N}$, followed by the neutral-neutral reaction of $\mathrm{N}$ and $\mathrm{CH}$, and dissociative recombination of $\mathrm{HCNH}^{+}$ at later times $\left(t \gtrsim 10^{4} \mathrm{yr}\right)$. Other, less important formation pathways for $\mathrm{CN}$ at this stage are neutral-neutral reactions between $\mathrm{C}$ and $\mathrm{NO}$ producing $\mathrm{CN}$ and $\mathrm{O}$, as well as between $\mathrm{C}$ and $\mathrm{OCN}$, leading to $\mathrm{CN}$ and $\mathrm{CO}$. The destruction of $\mathrm{CN}$ mostly proceeds through photodissociation and neutral-neutral reaction with $\mathrm{N}$ forming highly photostable nitrogen molecules and $\mathrm{C}$.

In turn, $\mathrm{HCNH}^{+}$is produced by ion-neutral reactions between $\mathrm{H}_{2}$ and $\mathrm{HCN}^{+}$and between $\mathrm{N}$ and $\mathrm{C}_{n} \mathrm{H}_{2}^{+}(n=3,4)$ at early times $\left(t \lesssim 10^{3} \mathrm{yr}\right)$. Later the steady state recycling of $\mathrm{HCNH}^{+}$is reached, which involves dissociative recombination of $\mathrm{HCNH}^{+}$ into either $\mathrm{HCN}(33 \%)$, HNC (33\%), or CN (33\%), followed by reproduction of $\mathrm{HCNH}^{+}$via protonation of $\mathrm{HCN}$ and $\mathrm{HNC}$ by either $\mathrm{H}_{3}^{+}, \mathrm{H}_{3} \mathrm{O}^{+}$, or $\mathrm{HCO}^{+}$as well as via the ion-neutral reaction of $\mathrm{CN}$ with $\mathrm{H}_{3}^{+}$.

The chemistry of $\mathrm{HCN}$ is tightly related to the evolution of $\mathrm{CN}$ and $\mathrm{HCNH}^{+}$. $\mathrm{HCN}$ is mainly produced by neutral-neutral reactions involving nitrogen and methylene or methyl at early evolutionary stages, $t \lesssim 10^{4} \mathrm{yr}$. Later the evolution of HCN proceeds as a part of the $\mathrm{HCNH}^{+}$formation-destruction loop. The major destruction routes for gas-phase HCN include freezeout onto dust grains and the ion-neutral reaction with $\mathrm{C}^{+}$, which forms $\mathrm{CNC}^{+}$and $\mathrm{H}$. Another important destruction channel for $\mathrm{HCN}$ in the upper disk layers at $\sim 1.5-2$ scale heights is photodissociation. Steady state for $\mathrm{HCN}$ is typically reached within $\gtrsim 10^{4} \mathrm{yr}$, with an abundance of about a few $\times 10^{-10}$.

The layer where abundant ammonia exists is located somewhat deeper toward the disk midplane compared with $\mathrm{HCN}$ and especially $\mathrm{CN}$ because $\mathrm{NH}_{3}$ can be photodissociated by UV photons shortward of $\approx 1950 \AA$ (van Dishoeck 1988). The main formation route for ammonia is dissociative recombination of $\mathrm{NH}_{4}^{+}$, which itself is formed through a sequence of hydrogen insertion reactions starting with the production of the $\mathrm{NH}^{+}$ions from $\mathrm{N}^{+}$reacting with $\mathrm{H}_{2}$. On formation, $\mathrm{NH}^{+}$repeatedly reacts with molecular hydrogen and gains an addition hydrogen atom until $\mathrm{NH}_{4}^{+}$is formed. The destruction of ammonia proceeds mainly via ion-neutral reactions with $\mathrm{C}^{+}$and $\mathrm{S}^{+}$in the upper intermediate layer, as well as $\mathrm{H}_{3}^{+}, \mathrm{H}_{3} \mathrm{O}^{+}$, and $\mathrm{HCO}^{+}$in a deeper region, which leads again to $\mathrm{NH}_{4}^{+}$and either $\mathrm{H}_{2}, \mathrm{H}_{2} \mathrm{O}$, or $\mathrm{CO}$. For all radii, the abundance of ammonia reaches a steady state value of $\lesssim 3 \times$ $10^{-10}$ to $3 \times 10^{-9}$ at very late times of $\gtrsim 10^{5}-10^{6} \mathrm{yr}$.

The chemical evolution of CS is governed by neutral-neutral reactions between sulfur atoms and either $\mathrm{CH}, \mathrm{CH}_{2}$, or $\mathrm{C}_{2}$ ini-

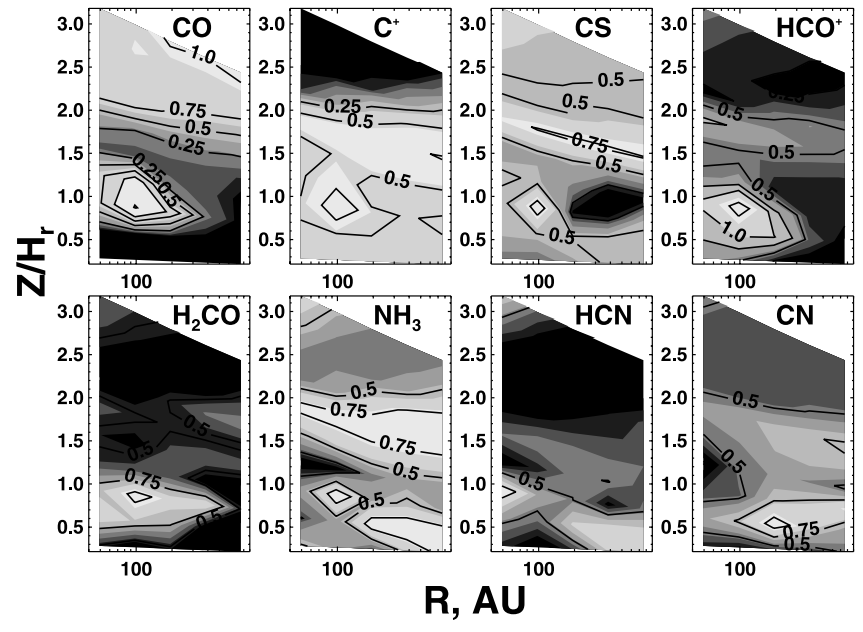

FIG. 4.- Same as in Fig. 3 but for abundance uncertainties (logarithmic units). [See the electronic edition of the Journal for a color version of this figure.]

tially, and later at $t \gtrsim 10^{3} \mathrm{yr}$, by an extended formation-destruction cycle of $\mathrm{H}_{n} \mathrm{CS}^{+}(n=1-3)$ and $\mathrm{HC}_{2} \mathrm{~S}^{+}$. At this evolutionary stage these complex ions are produced by ion-neutral reactions of protonated water, $\mathrm{H}_{3}^{+}$, and $\mathrm{HCO}^{+}$with $\mathrm{H}_{n} \mathrm{CS}(n=0-2)$. The initial routes to form the $\mathrm{HCS}^{+}$ion are the reactions between $\mathrm{CH}_{3}^{+}$ and $\mathrm{S}$ or $\mathrm{CS}^{+}$and $\mathrm{H}_{2}$, whereas $\mathrm{HC}_{2} \mathrm{~S}^{+}$forms predominantly via reaction of $\mathrm{CH}_{3}$ and $\mathrm{S}^{+}$. The $\mathrm{H}_{3} \mathrm{CS}^{+}$ion is mostly produced in reactive collisions of methane with sulfur ions. On formation, $\mathrm{HCS}^{+}$dissociatively recombines into $\mathrm{CS}(\sim 10 \%)$ or $\mathrm{CH}(\sim 90 \%)$, while $\mathrm{HC}_{2} \mathrm{~S}^{+}$breaks up equally into $\mathrm{C}_{2} \mathrm{~S}$ or $\mathrm{CS}$. The $\mathrm{H}_{2} \mathrm{CS}^{+}$and $\mathrm{H}_{3} \mathrm{CS}^{+}$ions recombine with equal probabilities into CS and either $\mathrm{HCS}$ or $\mathrm{H}_{2} \mathrm{CS}$. Finally, the major destruction channels for $\mathrm{CS}$ are photodissociation and the ion-neutral reaction with $\mathrm{C}^{+}$. The steady state abundance for CS, $\sim 10^{-9}$, is usually attained at about $10^{3}-10^{5}$ yr in our model.

\subsection{Distribution of the Abundance and Column Density Uncertainties}

For each key molecule, we derived the distribution of abundance uncertainties (dispersion) in the disk. The results are shown in Figure 4. These uncertainties are quite different in different disk regions. Note that the overall abundance uncertainties are not much larger for the more complex (heavy) species among those considered, in comparison to those with fewer atoms (e.g., compare $\mathrm{C}^{+}, \mathrm{CO}, \mathrm{HCO}^{+}$, and $\mathrm{H}_{2} \mathrm{CO}$ ). However, if we focused on even more complex species (e.g., consisting of $>7$ atoms), the abundance uncertainties will in general increase with the number of atoms in the molecule, as shown in Paper I and Wakelam et al. (2006a).

Among the key reactions, the rates of photodissociation and photoionization processes can be uncertain by factors of 2 and larger. Moreover, their absolute values depend on the details of the UV radiative transfer modeling (van Zadelhoff et al. 2003). Thus, it is not surprising that in the disk atmosphere at $Z / H(r) \gtrsim 1.5-2$ large abundance uncertainties with a factor of $\$ 10-30$ are reached for most of the species, in particular across the interface between the intermediate layer and the disk atmosphere. A notable exception is ionized carbon, because it contains essentially all of the element carbon in the disk atmosphere and thus maintains a high steady state abundance that is not affected by chemistry. This effect leads to relatively low values of the $\mathrm{C}^{+}$abundance uncertainties, in particular a factor of $\lesssim 3$. The same is true for $\mathrm{CO}$, which locks up all carbon in the disk midplane and intermediate layer at $\$ 2$ pressure scale height 




FIG. 5.-Same as in Fig. 4 but for radial distributions of the column densities. The central solid line represents the model-averaged column densities. The corresponding column density scatters computed with the standard model are depicted with dotted lines. The column density scatters calculated with the chemical model where the rates of the 56 most problematic reactions are varied by small factors of 1.25-2 only are shown with solid lines.

and here has its lowest abundance uncertainty, a factor of $\lesssim 2$ (Fig. 4).

There is a disk region adjacent to the midplane at $r \sim 100 \mathrm{AU}$, $Z / H(r) \sim 1$, where the abundance uncertainties of the considered carbon-bearing species including $\mathrm{CO}$ show an increase of up to one order of magnitude (see $\mathrm{HCO}^{+}$; Fig. 4). There, the chemistry of $\mathrm{CO}$ and hence all chemically related species (e.g., $\mathrm{HCO}^{+}, \mathrm{H}_{2} \mathrm{CO}$ ) are prone to the hypersensitivity at $\gtrsim 10^{6} \mathrm{yr}$ caused by the rate uncertainty of the X-ray ionization of helium atoms, as discussed in Wakelam et al. (2006b). This region is moderately warm, with a temperature $T \sim 25-40 \mathrm{~K}$, and well shielded from the UV radiation from the central star and the interstellar UV radiation $\left(A_{V} \lesssim 2-5 \mathrm{mag}\right)$. The thermal bremsstrahlung X-ray photons $(\sim 5 \mathrm{keV})$ generated at $\sim 0.1 \mathrm{AU}$ above the star, however, are able to penetrate into this region (Glassgold et al. 1997a), with a total ionization rate of $\sim 10^{-16} \mathrm{~s}^{-1}$. These X-ray photons produce helium ions that rapidly react and destroy the $\mathrm{CO}$ molecules (forming $\mathrm{O}$ and $\mathrm{C}^{+}$). In turn, these $\mathrm{C}^{+}$ions are used to reform $\mathrm{CO}$ but also to slowly produce carbon chain molecules (e.g., $\mathrm{C}_{2} \mathrm{H}_{2}$ ) and cyanopolyynes (e.g., $\mathrm{HC}_{3} \mathrm{~N}$ ), which are removed from the gas phase by freezeout onto the dust surfaces. These heavy surface molecules lock up most of the elemental carbon at $t \gtrsim 1 \mathrm{Myr}$ such that the gas-phase abundance of $\mathrm{CO}$ is reduced to $\sim 10^{-9}$ to $10^{-7}$. The overall efficiency of the carbon chain formation and the decline of the $\mathrm{CO}$ abundance at late times sensitively depend on the He ionization rate. Consequently, the $\mathrm{CO}$ histogram of the abundance distribution at $5 \mathrm{Myr}$ has a doublepeaked, non-Gaussian shape, as shown in Figure 2.

The abundances of nitrogen-bearing species such as $\mathrm{CN}, \mathrm{HCN}$, and $\mathrm{NH}_{3}$ in the region around the midplane at $r>200 \mathrm{AU}$ (Fig. 4) possesses a high sensitivity to the rate of the CRP ionization of He. The histograms of $\mathrm{CN}, \mathrm{HCN}$, and $\mathrm{NH}_{3}$ are not fully Gaussian at $5 \mathrm{Myr}$ in this region, and our method tends to overestimate the resulting abundance uncertainties. This region is so cold that at late times, $t \gtrsim 3 \times 10^{5} \mathrm{yr}$, most molecules are removed from the gas phase by freezeout. At this evolutionary stage, $\mathrm{H}_{3}^{+}$and later $\mathrm{H}^{+}$become the most abundant charged species. The primal formation pathway for $\mathrm{H}^{+}$is the slow ion-neutral reaction between $\mathrm{He}^{+}$and $\mathrm{H}_{2}$. The rise of the $\mathrm{H}^{+}$abundance at $\sim 5 \mathrm{Myr}\left(\lesssim 10^{-8}\right)$ occurs in part because of the slowness of the radiative recom-
TABLE 2

Typical Column Density Uncertainties

\begin{tabular}{|c|c|c|}
\hline Species & Standard Model & Improved Model \\
\hline $\mathrm{H}_{3}^{+}$ & $\sim 2$ & $\sim 1.5$ \\
\hline 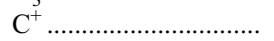 & $\sim 2$ & $\sim 1.5$ \\
\hline $\mathrm{CO}$ & $\sim 1.25$ & 1.1 \\
\hline $\mathrm{CO}_{2} \ldots \ldots \ldots \ldots \ldots \ldots$ & $\sim 3.5$ & $\sim 2.5$ \\
\hline 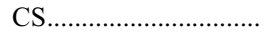 & 3 & 2.5 \\
\hline 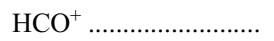 & $\sim 3$ & $\sim 1.5$ \\
\hline $\mathrm{H}_{2} \mathrm{CO}$ & 3 & $\sim 1.5$ \\
\hline $\mathrm{CN}$ & 4 & 2 \\
\hline HCN & $\sim 3$ & 2.5 \\
\hline HNC …………….... & 3 & $\sim 2.5$ \\
\hline $\mathrm{N}_{2} \mathrm{H}^{+} \ldots \ldots \ldots \ldots \ldots \ldots \ldots$ & 2.5 & 2 \\
\hline $\mathrm{NH}_{3} \ldots \ldots \ldots \ldots \ldots \ldots \ldots$ & 2.5 & 2.5 \\
\hline 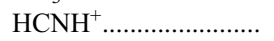 & 2.5 & 2.5 \\
\hline $\mathrm{H}_{2} \mathrm{O}$ & 1.7 & 1.4 \\
\hline $\mathrm{C}_{2} \mathrm{H}$ & 4 & $\sim 3$ \\
\hline
\end{tabular}

NotE.-Standard Model and Improved Model list factor values.

bination of ionized hydrogen atoms in the molecular-deficient gas. The total electron concentration increases in tandem with $\mathrm{H}^{+}$ and leads to more rapid dissociative recombination of $\mathrm{HCNH}^{+}$ and $\mathrm{NH}_{4}^{+}$(precursor ions for $\mathrm{CN}, \mathrm{HCN}$, and $\mathrm{NH}_{3}$ ). Consequently, the previously low gas-phase abundances of these nitrogenbearing molecules increase at $t \sim 5 \mathrm{Myr}$ by factors of several. The steepness and evolutionary pattern for these abundance gradients depend sensitively on the abundance of helium ions.

In the rest of the intermediate layer, the chemical evolution of the gas-phase species is initiated by radiative association reactions with high uncertainties but governed later by ion-neutral and neutral-neutral reactions with many accurately estimated rates. The situation in the disk midplane is similar, although at later times the chemical evolution of many gas-phase species is governed by the steady state accretion-desorption life cycle, which has no uncertainties in our model. This effect leads to abundance uncertainties in the intermediate layer that have values that are lower than a factor of 3 and thus are lower than the abundance uncertainties in the upper disk region (see Fig. 4).

It is interesting to note that for $\mathrm{CO}, \mathrm{C}^{+}$, and $\mathrm{NH}_{3}$ the uncertainty peak does not coincide with the maximal relative abundance in the disk. One might expect that the scatter in their column densities could be smaller than for other considered species (CS, $\mathrm{CN}, \mathrm{HCN}, \mathrm{HCO}^{+}$, and $\mathrm{H}_{2} \mathrm{CO}$ ), for which uncertainty peaks and maximum abundances tend to coincide.

In Figure 5, the radial distributions of the column densities at $5 \mathrm{Myr}$ are plotted for the considered species (dotted lines). In addition, in Table 2 we compile typical intrinsic uncertainties of the column densities of a larger set of key molecules in protoplanetary disks at $100 \mathrm{AU}$. Indeed, the overall uncertainty in the column densities of $\mathrm{CO}, \mathrm{C}^{+}$, and $\mathrm{NH}_{3}$ as well as $\mathrm{H}_{3}^{+}, \mathrm{H}_{2} \mathrm{O}, \mathrm{N}_{2} \mathrm{H}^{+}$, and $\mathrm{HCNH}^{+}$is less than a factor of about 2. Using this uncertainty value as a criterion, we assign these species to the so-called first uncertainty group. On the other hand, the column densities of $\mathrm{CS}, \mathrm{CN}, \mathrm{HCN}, \mathrm{HCO}^{+}$, and $\mathrm{H}_{2} \mathrm{CO}$ as well as $\mathrm{CO}_{2}, \mathrm{C}_{2} \mathrm{H}$, and $\mathrm{HNC}$ have larger error bars up to a factor of 4 . These species belong to the second uncertainty group. It is likely that the species from the first sensitivity group are more reliable observational tracers than the species from the second one.

Note that the column density uncertainties do not exceed a factor of about 4 even for formaldehyde and are comparable with observational uncertainties. However, for heavier species (e.g., $\mathrm{HC}_{9} \mathrm{~N}$ ), which are not included in our study, the uncertainties are 
likely larger, as shown in Paper I and Paper II. In our model, these uncertainties slowly increase with time and typically reach steady state at $\gtrsim 10^{5} \mathrm{yr}$. The column density uncertainties can be used as error bars of the theoretically predicted quantities when comparing with observational data. Moreover, one can envisage a situation when, for an object with a well-studied structure, highresolution observations followed by advanced modeling will allow putting such tight constraints on molecular abundances or column densities that it may help to determine the rate coefficients of some key reactions observationally.

\section{DISCUSSION}

In the previous section, we demonstrated how and to what extent the uncertainties of the rate coefficients affect the computed abundances and column densities of several key species in the disk. In this section, we discuss the relative contributions of individual reactions to the uncertainties of these key molecules.

\subsection{Sensitivity Analysis Method}

The chemical evolution of even a simple molecule usually involves a large number of reactions in the adopted chemical network. The nonlinearity of the equations of chemical kinetics often makes it a challenge to find a direct link between the rate value of a certain reaction and the molecular abundances. Thus an efficient numerical method is required (see, e.g., Turanyi 1997).

Using a sensitivity analysis based on a simple correlation method, we showed in Paper I that under the physical conditions of dark and diffuse clouds the rate uncertainties of only a handful of chemical reactions affect the accuracy of the resulting abundances to the highest extent. For the disk chemistry studied in this paper a modified, two-stage sensitivity analysis was performed.

First, for each selected molecule, we calculated time-dependent linear correlation coefficients between the abundances and rate coefficients for all 8000 realizations of the RATE 06 network, and 19 logarithmically taken time steps over the $5 \mathrm{Myr}$ of disk evolution at each disk cell. The coefficients $R_{i j}(t)$ are given by the expression

$$
R_{i j}(r, z, t)=\frac{\sum_{l}\left[x_{i}^{l}(r, z, t)-\overline{x_{i}(r, z, t)}\right]\left(\alpha_{j}^{l}-\overline{\alpha_{j}}\right)}{\sqrt{\sum_{l}\left[x_{i}^{l}(r, z, t)-\overline{x_{i}(r, z, t)}\right]^{2} \sum_{l}\left(\alpha_{j}^{l}-\overline{\alpha_{j}}\right)^{2}}},
$$

where $x_{i}^{l}(r, z, t)$ is the molecular abundance of the $i$ th species computed with the $l$ th realization of the UMIST network, $\overline{x_{i}(r, z, t)}$ is the standard (mean) abundance of this species, $\alpha_{j}^{l}$ is the $j$ th reaction rate coefficient for the $l$ th realization of the RATE 06 network, and $\overline{\alpha_{j}}$ is the standard rate coefficient of the $j$ th reaction.

This is in contrast to Paper I where the analysis of statistically significant reactions was performed for only the final time step. Since at various evolutionary stages the chemistry of a molecule is typically governed by a restricted number of chemical processes, at any particular time step the abundance correlation will be high only for some of the key reactions, as shown in Figure 6 for the case of ammonia. The obvious drawback of such a "single-time" approach is that it does not account for the cumulative character of the abundance uncertainties. Therefore, we integrated the absolute values of time-dependent correlation coefficients over the entire evolutionary time of $5 \mathrm{Myr}$. These quantities are called cumulative correlation coefficients.

With this cumulative criterion, one can identify those reactions whose rate uncertainties are the most important for the abundance scatter of one molecule in one specific disk region. As a general criterion for the entire disk, we utilized the cumulative correlation

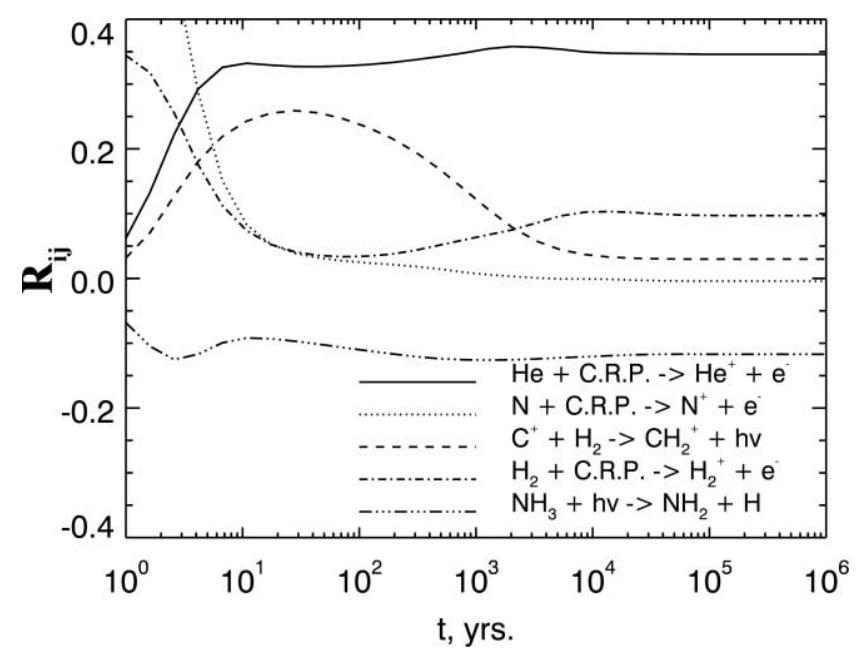

FIG. 6.- Largest correlation coefficients of relevant reactions for the evolution of $\mathrm{NH}_{3}$ as a function of time in the disk intermediate layer $(r=97 \mathrm{AU}, z=30 \mathrm{AU})$.

summed up over all 50 disk grid cells, followed by summation over the eight considered species $\left(\mathrm{CO}, \mathrm{C}^{+}, \mathrm{CS}, \mathrm{HCO}^{+}, \mathrm{H}_{2} \mathrm{CO}\right.$, $\mathrm{CN}, \mathrm{HCN}, \mathrm{NH}_{3}$ ). One should bear in mind that the results of our analysis rely on the inherent assumption that the absolute values of the rate coefficients and their uncertainties in RATE 06 are accurately determined. This assumption may not be fulfilled for all important reactions in the RATE 06 database as we will show below. Such reactions will not be treated correctly by our sensitivity analysis.

\subsection{Identification of the Most Problematic Reactions}

We calculated disk-averaged cumulative correlations for all 5773 reactions in our chemical network and for eight key species: $\mathrm{CO}, \mathrm{C}^{+}, \mathrm{CS}, \mathrm{HCO}^{+}, \mathrm{H}_{2} \mathrm{CO}, \mathrm{CN}, \mathrm{HCN}$, and $\mathrm{NH}_{3}$. The corresponding cumulative correlations, normalized in descending order, steadily decline with the number of reactions. The 56 most problematic reactions and their weights for these eight molecules are listed in Table 3. We term by "weight" the corresponding cumulative correlations summed over the all 50 disk cells and renormalized to the maximum value of 1148.1, which is achieved for the radiative association (RA) reaction between $\mathrm{H}_{2}$ and C.

These 56 reactions correspond to $1 \%$ of the total number of reactions in our chemical model. Moreover, such an apparently small number of reactions is chosen to stimulate experimental and theoretical studies of the rate coefficients of the reactions with high astrochemical importance. Note also that some of the most problematic reactions in Table 3 have been carefully studied as their rates have small uncertainties of only factors of 1.25-1.5.

In order to demonstrate that these 56 reactions are indeed important and their rate uncertainties strongly affect the abundances, we recalculated the disk chemical evolution but allowed the rates of these 56 reactions to vary by only a factor of 1.25 (and 2 for radiative association). Such idealization is thought to mimic the situation when all of these 56 rates will be accurately measured in the laboratory or derived theoretically.

The resulting uncertainties in the molecular column densities are shown in Figure 5 (solid lines) and reported in Table 3 (last column). Compared with the initial column density uncertainties, the refined chemical model leads to smaller error bars for most of the considered molecules, in particular for $\mathrm{CO}, \mathrm{HCO}^{+}, \mathrm{H}_{2} \mathrm{CO}$, $\mathrm{CN}$, and $\mathrm{H}_{2} \mathrm{O}$. However, the improvement is not so obvious for nitrogen-bearing species $\left(\mathrm{HCN}, \mathrm{HNC}, \mathrm{NH}_{3}, \mathrm{HCNH}^{+}\right.$) or for $\mathrm{CO}_{2}, \mathrm{CS}$, and $\mathrm{C}_{2} \mathrm{H}$. Their chemical evolution is governed by a 
TABLE 3

Most Problematic Reactions

\begin{tabular}{|c|c|c|c|}
\hline Reaction & Uncertainty $^{\mathrm{a}}$ & Weight & Type \\
\hline $\mathbf{H}_{2}+\mathbf{C} \rightarrow \mathbf{C H}_{2}+\boldsymbol{h} \nu \ldots \ldots \ldots \ldots \ldots \ldots$ & 10 & 1.0 & $\mathbf{R A}$ \\
\hline $\mathbf{H}_{2}+\mathbf{C}^{+} \rightarrow \mathbf{C H}_{2}^{+}+\boldsymbol{h} \nu \ldots \ldots \ldots \ldots \ldots$ & 10 & $4.2(-1)$ & RA \\
\hline $\mathrm{H}_{2}+\mathrm{CH} \rightarrow \mathrm{CH}_{3}+h \nu \ldots \ldots \ldots \ldots \ldots \ldots$ & 10 & $1.6(-2)$ & RA \\
\hline $\mathrm{H}_{2}+\mathrm{CH}_{3}^{+} \rightarrow \mathrm{CH}_{5}^{+}+h \nu \ldots \ldots \ldots \ldots \ldots$ & 10 & $7.6(-2)$ & RA \\
\hline $\mathrm{C}+\mathrm{H} \rightarrow \mathrm{CH}+h \nu \ldots \ldots \ldots \ldots \ldots \ldots \ldots$ & 10 & $2.3(-2)$ & RA \\
\hline $\mathrm{C}+\mathrm{N} \rightarrow \mathrm{CN}+h \nu \ldots \ldots$ & 10 & $2.2(-2)$ & RA \\
\hline $\mathrm{C}^{+}+e^{-} \rightarrow \mathrm{C}+h \nu \ldots \ldots$ & 10 & $1.2(-2)$ & $\mathrm{RR}$ \\
\hline $\mathrm{CH}_{3}^{+}+e^{-} \rightarrow \mathrm{CH}_{3}+h \nu \ldots \ldots \ldots \ldots \ldots$ & 10 & $1.2(-2)$ & RR \\
\hline $\mathbf{H}_{2}+$ C.R.P. $\rightarrow \mathbf{H}_{2}^{+}+\boldsymbol{e}^{-} \ldots \ldots \ldots \ldots \ldots$ & $2^{\mathrm{b}}$ & $1.4(-1)$ & CRI \\
\hline $\mathrm{H}_{2}+$ C.R.P. $\rightarrow \mathrm{H}^{+}+\mathrm{H}+e^{-} \ldots \ldots \ldots$ & 2 & $1.0(-2)$ & CRI \\
\hline $\mathrm{He}+$ C.R.P. $\rightarrow \mathrm{He}^{+}+e^{-} \ldots \ldots \ldots \ldots \ldots$ & 2 & $2.3(-1)$ & CRI \\
\hline $\mathrm{N}+$ C.R.P. $\rightarrow \mathrm{N}^{+}+e^{-} \ldots \ldots \ldots \ldots \ldots \ldots$ & 2 & $9.7(-2)$ & CRI \\
\hline $\mathrm{C}+h \nu \rightarrow \mathrm{C}^{+}+e^{-} \ldots \ldots \ldots \ldots \ldots \ldots \ldots \ldots \ldots \ldots \ldots$ & 2 & $3.8(-1)$ & PI \\
\hline $\mathrm{C}+$ C.R.P. $h \nu \rightarrow \mathrm{C}^{+}+e^{-} \ldots \ldots \ldots \ldots \ldots$ & 2 & $6.7(-2)$ & PI \\
\hline $\mathrm{CO}+h \nu \rightarrow \mathrm{O}+\mathrm{C} \ldots \ldots \ldots \ldots \ldots \ldots \ldots \ldots \ldots \ldots \ldots$ & 10 & $5.6(-2)$ & $\mathrm{PD}$ \\
\hline $\mathrm{CH}+h \nu \rightarrow \mathrm{C}+\mathrm{H} \ldots \ldots \ldots \ldots \ldots \ldots \ldots \ldots \ldots \ldots \ldots \ldots \ldots$ & 2 & $2.3(-2)$ & PD \\
\hline $\mathrm{CH}_{2}+h \nu \rightarrow \mathrm{CH}+\mathrm{H} \ldots \ldots \ldots \ldots \ldots \ldots$ & 2 & $6.8(-3)$ & $\mathrm{PD}$ \\
\hline $\mathrm{CN}+h \nu \rightarrow \mathrm{N}+\mathrm{C} \ldots \ldots \ldots \ldots$ & 2 & $3.2(-2)$ & PD \\
\hline $\mathrm{CS}+h \nu \rightarrow \mathrm{S}+\mathrm{C} \ldots \ldots \ldots \ldots \ldots$ & 2 & $2.6(-2)$ & $\mathrm{PD}$ \\
\hline $\mathrm{NH}_{3}+h \nu \rightarrow \mathrm{NH}_{2}+\mathrm{H} \ldots \ldots \ldots \ldots \ldots . . . . .$. & 1.5 & $8.2(-3)$ & PD \\
\hline $\mathrm{HCN}+h \nu \rightarrow \mathrm{CN}+\mathrm{H} \ldots \ldots \ldots \ldots \ldots \ldots$ & 1.5 & $1.5(-2)$ & $\mathrm{PD}$ \\
\hline $\mathrm{OH}+h \nu \rightarrow \mathrm{O}+\mathrm{H} \ldots \ldots \ldots \ldots \ldots \ldots \ldots \ldots$ & 1.5 & $7.8(-3)$ & PD \\
\hline $\mathrm{He}^{+}+\mathrm{CO} \rightarrow \mathrm{O}+\mathrm{C}^{+}+\mathrm{He} \ldots \ldots \ldots \ldots$ & 1.25 & $9.6(-3)$ & IN \\
\hline $\mathrm{He}^{+}+\mathrm{N}_{2} \rightarrow \mathrm{N}+\mathrm{N}^{+}+\mathrm{He} \ldots \ldots \ldots \ldots$ & 1.25 & $7.4(-3)$ & $\mathrm{IN}$ \\
\hline $\mathrm{H}_{3}^{+}+\mathrm{C} \rightarrow \mathrm{CH}^{+}+\mathrm{H}_{2} \ldots \ldots \ldots \ldots \ldots \ldots \ldots$ & 2 & $3.3(-2)$ & IN \\
\hline $\mathrm{H}_{3}^{+}+\mathrm{O} \rightarrow \mathrm{OH}^{+}+\mathrm{H}_{2} \ldots \ldots \ldots \ldots \ldots \ldots$ & 1.5 & $1.0(-2)$ & IN \\
\hline $\mathrm{H}_{3}^{+}+\mathrm{CO} \rightarrow \mathrm{HCO}^{+}+\mathrm{H}_{2} \ldots \ldots \ldots \ldots \ldots$ & 1.25 & $7.3(-3)$ & IN \\
\hline $\mathrm{H}_{2}^{+}+\mathrm{N} \rightarrow \mathrm{NH}^{+}+\mathrm{H} \ldots \ldots \ldots \ldots \ldots \ldots \ldots$ & 2 & $4.3(-2)$ & IN \\
\hline $\mathrm{H}_{2}+\mathrm{He}^{+} \rightarrow \mathrm{He}+\mathrm{H}^{+}+\mathrm{H} \ldots \ldots \ldots \ldots$ & 2 & $2.0(-2)$ & IN \\
\hline $\mathrm{H}_{2}+\mathrm{He}^{+} \rightarrow \mathrm{He}+\mathrm{H}_{2}^{+} \ldots \ldots \ldots \ldots \ldots \ldots$ & 2 & $2.1(-2)$ & IN \\
\hline $\mathrm{H}_{2}+\mathrm{NH}_{3}^{+} \rightarrow \mathrm{NH}_{4}^{+}+\mathrm{H} \ldots \ldots \ldots \ldots \ldots$ & 1.5 & $4.4(-2)$ & IN \\
\hline 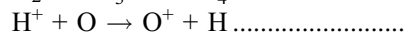 & 1.5 & $8.4(-3)$ & $\mathrm{CT}$ \\
\hline $\mathrm{C}^{+}+\mathrm{CH} \rightarrow \mathrm{C}_{2}^{+}+\mathrm{H} \ldots \ldots \ldots \ldots \ldots \ldots \ldots$ & 2 & $1.3(-2)$ & IN \\
\hline $\mathrm{C}^{+}+\mathrm{OH} \rightarrow \mathrm{CO}^{+}+\mathrm{H} \ldots \ldots \ldots \ldots \ldots \ldots$ & 2 & $3.1(-2)$ & IN \\
\hline $\mathrm{C}^{+}+\mathrm{NH} \rightarrow \mathrm{CN}^{+}+\mathrm{H} \ldots \ldots \ldots \ldots \ldots \ldots$ & 2 & $1.1(-2)$ & IN \\
\hline $\mathrm{C}^{+}+\mathrm{HCN} \rightarrow \mathrm{CNC}^{+}+\mathrm{H} \ldots .$. & 1.25 & $7.9(-3)$ & IN \\
\hline $\mathrm{C}+\mathrm{HCO}^{+} \rightarrow \mathrm{CO}+\mathrm{CH}^{+} \ldots \ldots$ & 2 & $2.7(-2)$ & IN \\
\hline $\mathrm{CH}+\mathrm{S}^{+} \rightarrow \mathrm{CS}^{+}+\mathrm{H} \ldots \ldots \ldots \ldots \ldots \ldots \ldots$ & 2 & $2.6(-2)$ & IN \\
\hline $\mathrm{CH}_{2}^{+}+\mathrm{O} \rightarrow \mathrm{HCO}^{+}+\mathrm{H} \ldots \ldots \ldots \ldots \ldots$. & 2 & $7.2(-3)$ & IN \\
\hline $\mathrm{NH}_{3}^{+}+\mathrm{Mg} \rightarrow \mathrm{NH}_{3}+\mathrm{Mg}^{+} \ldots \ldots \ldots \ldots$ & 2 & $1.1(-2)$ & $\mathrm{CT}$ \\
\hline $\mathrm{C}+\mathrm{CH}_{2} \rightarrow \mathrm{C}_{2} \mathrm{H}+\mathrm{H} \ldots \ldots \ldots \ldots \ldots \ldots$ & 2 & $3.6(-2)$ & $\mathrm{NN}$ \\
\hline $\mathrm{C}+\mathrm{C}_{2} \mathrm{H} \rightarrow \mathrm{C}_{3}+\mathrm{H} \ldots \ldots \ldots \ldots \ldots \ldots \ldots$ & 2 & $8.3(-3)$ & $\mathrm{NN}$ \\
\hline $\mathrm{N}+\mathrm{CH}_{2} \rightarrow \mathrm{HCN}+\mathrm{H} \ldots \ldots \ldots \ldots \ldots \ldots$ & 1.5 & $8.5(-2)$ & $\mathrm{NN}$ \\
\hline $\mathrm{N}+\mathrm{CN} \rightarrow \mathrm{N}_{2}+\mathrm{C} \ldots \ldots \ldots \ldots \ldots \ldots \ldots \ldots$ & 2 & $4.6(-2)$ & $\mathrm{NN}$ \\
\hline $\mathrm{N}+\mathrm{HCO} \rightarrow \mathrm{HCN}+\mathrm{O} \ldots \ldots \ldots \ldots \ldots$ & 2 & $2.9(-2)$ & $\mathrm{NN}$ \\
\hline $\mathrm{H}+\mathrm{CH}_{2} \rightarrow \mathrm{CH}+\mathrm{H}_{2} \ldots \ldots \ldots \ldots \ldots \ldots$ & 1.25 & $1.9(-2)$ & $\mathrm{NN}$ \\
\hline $\mathrm{H}+\mathrm{CH} \rightarrow \mathrm{C}+\mathrm{H}_{2} \ldots \ldots \ldots \ldots \ldots \ldots \ldots \ldots$ & 1.5 & $1.1(-2)$ & NN \\
\hline $\mathrm{CH}+\mathrm{O} \rightarrow \mathrm{HCO}^{+}+e^{-} \ldots \ldots \ldots \ldots \ldots \ldots$ & 1.5 & $4.0(-2)$ & $\mathrm{NN}$ \\
\hline $\mathrm{CH}+\mathrm{O} \rightarrow \mathrm{CO}+\mathrm{H} \ldots \ldots \ldots \ldots \ldots \ldots \ldots$ & 1.25 & $8.3(-3)$ & $\mathrm{NN}$ \\
\hline $\mathrm{CH}+\mathrm{N} \rightarrow \mathrm{CN}+\mathrm{H} \ldots \ldots \ldots \ldots \ldots \ldots \ldots$ & 1.25 & $2.3(-2)$ & $\mathrm{NN}$ \\
\hline $\mathrm{CH}+\mathrm{S} \rightarrow \mathrm{CS}+\mathrm{H} \ldots \ldots \ldots \ldots \ldots \ldots \ldots$ & 2 & $1.6(-2)$ & $\mathrm{NN}$ \\
\hline $\mathrm{CH}_{2}+\mathrm{S} \rightarrow \mathrm{CS}+\mathrm{H}_{2} \ldots \ldots \ldots \ldots \ldots \ldots \ldots$ & 2 & $6.3(-2)$ & NN \\
\hline $\mathrm{CH}_{2}+\mathrm{O} \rightarrow \mathrm{CO}+\mathrm{H}+\mathrm{H} \ldots \ldots \ldots \ldots \ldots$ & 1.25 & $1.0(-2)$ & $\mathrm{NN}$ \\
\hline $\mathrm{CH}_{3}+\mathrm{O} \rightarrow \mathrm{H}_{2} \mathrm{CO}+\mathrm{H} \ldots \ldots \ldots \ldots \ldots$ & 1.25 & $1.4(-2)$ & $\mathrm{NN}$ \\
\hline $\mathrm{HCO}^{+}+e^{-} \rightarrow \mathrm{CO}+\mathrm{H} \ldots \ldots \ldots \ldots \ldots . . . . . .$. & 1.25 & $3.1(-2)$ & DR \\
\hline $\mathrm{NH}_{4}^{+}+e^{-} \rightarrow \mathrm{NH}_{3}+\mathrm{H} \ldots \ldots \ldots \ldots \ldots \ldots$ & 1.25 & $8.1(-3)$ & DR \\
\hline
\end{tabular}

Notes.-_(CRI) Cosmic-ray ionization; (CT) charge transfer; (DR) dissociative recombination; ( IN) ion-neutral reaction; (NN) neutral-neutral reaction; (PD) photodissociation; (PI) photoionization; (RA) radiative association; (RR) radiative recombination. Three most important reactions for the disk chemistry as identified by the sensitivity analysis are written in boldface.

${ }^{a}$ Values in this column are factors of uncertainty.

b These rates are partly uncertain due to uncertainties in physical parameters. large set of key reactions that are only partly included in the list of the 56 most problematic reactions (Table 2 ). ${ }^{3}$

The fact that the uncertainties in column densities decrease not only for the eight key species, but also for some other molecules in Table 2 clearly indicates that some of the reactions from Table 3 are relevant for their chemical evolution. For $\mathrm{H}_{3}^{+}$these are the ionization of $\mathrm{H}_{2}$ and reactions of $\mathrm{H}_{3}^{+}$with $\mathrm{C}, \mathrm{O}$, and $\mathrm{CO}$. As we have shown in $\S 3.1$, reactions with $\mathrm{H}_{3}^{+}$are an essential ingredient of disk chemistry. The decrease in the abundance error bars for $\mathrm{H}_{3}^{+}$in the model with refined rate uncertainties leads to smaller abundance uncertainties in the related species: $\mathrm{HCO}^{+}, \mathrm{H}_{2} \mathrm{CO}$, $\mathrm{H}_{2} \mathrm{O}, \mathrm{HCN}, \mathrm{HNC}, \mathrm{HCNH}^{+}$, and CS. Table 3 contains a number of reactions important for the evolution of $\mathrm{CO}$ and $\mathrm{CO}_{2}$, e.g., formation of light hydrocarbons, neutral-neutral reactions of these hydrocarbons with oxygen, and $\mathrm{CO}$ photodissociation. The former reactions affect the evolution of $\mathrm{C}_{2} \mathrm{H}$, while photodissociation is an important process for $\mathrm{CN}$. Below we discuss the contributions of each reaction type in detail.

\subsubsection{Radiative Association}

The most problematic reactions in the disk are RA reactions. Radiative association leads to the formation of a larger molecule from two smaller species on their collision, and requires the emission of excess energy in the form of a photon. RA reactions allow the formation of new bonds and more chemically "advanced" species (van Dishoeck \& Blake 1998). The corresponding rate values are often so low for small species that they are difficult to obtain with high accuracy (Williams 1972; Herbst 1980; Bates \& Herbst 1988; Smith 1989). Nonetheless, assigned uncertainties for many of these reactions in RATE 06 are only a factor of 2 and less. Since we assume that the rate coefficients of such reactions may vary by an order of magnitude, in accord with the recent OSU database, it is not surprising that some RA reactions are identified as among the most problematic reactions for disk chemistry. Its importance also rises due to the fact that we start calculation from the atomic initial abundances and use time-integrated correlation coefficients. It means that at early evolutionary time chemistry is dominated by RA reactions, and therefore its time-integrated correlation coefficients increase greatly at early times.

Two RA reactions are particularly important for the chemical evolution and accumulation of the abundance uncertainties. First is the reaction between $\mathrm{H}_{2}$ and $\mathrm{C}$, which produces $\mathrm{CH}_{2}$ everywhere through the disk at all times. The rate coefficient of this reaction is not well known, with an estimated value of $10^{-17} \mathrm{~cm}^{3} \mathrm{~s}^{-1}$ at $T=10-300 \mathrm{~K}$ (Prasad \& Huntress 1980; Woodall et al. 2007). This reaction is relevant for the evolution of $\mathrm{CO}, \mathrm{HCO}^{+}, \mathrm{H}_{2} \mathrm{CO}$, $\mathrm{CS}$, and $\mathrm{HCN}$. Second, the reaction between $\mathrm{H}_{2}$ and $\mathrm{C}^{+}$leads to the formation of $\mathrm{CH}_{2}^{+}$(important for $\mathrm{C}^{+}, \mathrm{HCO}^{+}, \mathrm{CS}, \mathrm{NH}_{3}$, and $\mathrm{CN}$ ), with an estimated rate coefficient of $\approx 4 \times 10^{-17} \mathrm{~cm}^{3} \mathrm{~s}^{-1}$ (Herbst 1985). These two reactions initiate processes of $\mathrm{C}$ and $\mathrm{H}$ addition in the chemical model and thus should be relevant for nearly all species containing carbon and hydrogen.

The next most important RA reaction (8th place out of 56) is the formation of protonated methane from molecular hydrogen and $\mathrm{CH}_{3}^{+}\left(\alpha=1.3 \times 10^{-14} \mathrm{~cm}^{3} \mathrm{~s}^{-1}, \beta=-1\right.$; Smith 1989), which is an important molecular ion for $\mathrm{CO}$ destruction. More recently the rate of this reaction has been measured at low temperature of $10 \mathrm{~K}$ by Gerlich $(1995)(1.1 \pm 0.1) \times 10^{-13} \mathrm{~cm}^{3} \mathrm{~s}^{-1}$ for para- $\mathrm{H}_{2}$.

Much less important (27th and 28th place) are two slow reactions between $\mathrm{C}$ and $\mathrm{H}$ or $\mathrm{N}$ producing $\mathrm{CH}$ or $\mathrm{CN}$ radicals. It is interesting to note that the latter reaction has different rate

\footnotetext{
${ }^{3}$ An extended version of this table is freely available from the authors on request.
} 
coefficients in the OSU and RATE 06 databases (Smith et al. 2004; Woodall et al. 2007). While in osu.2007 this reaction rate has no temperature dependence and no activation barrier $(\alpha=$ $10^{-17} \mathrm{~cm}^{3} \mathrm{~s}^{-1}$; Prasad \& Huntress 1980), in RATE 06 the corresponding rate is low, $7.9 \times 10^{-19} \mathrm{~cm}^{3} \mathrm{~s}^{-1}$, and possesses a small barrier of $96 \mathrm{~K}$ (relevant temperature range is between 300 and $2700 \mathrm{~K}$; Singh \& Andreazza 2000). Thus the RATE 06 and osu.2007 rate coefficients for the RA reaction between C and N differ by an order of magnitude in a wide temperature range.

The situation is similar for the RA reaction between molecular hydrogen and $\mathrm{CH}$ that forms $\mathrm{CH}_{3}$ - an important radical for the evolution of formaldehyde. The corresponding osu.2007 rate at $10 \mathrm{~K}$ is $2.5 \times 10^{-16} \mathrm{~cm}^{3} \mathrm{~s}^{-1}\left(\alpha=3.25 \times 10^{-17} \mathrm{~cm}^{3} \mathrm{~s}^{-1}, \beta=\right.$ -0.6 ). In contrast, in RATE 06 this reaction has a small barrier of $11.6 \mathrm{~K}$ and the $10 \mathrm{~K}$ rate value is lower by an order of magnitude, $1.8 \times 10^{-17} \mathrm{~cm}^{3} \mathrm{~s}^{-1}\left(\alpha=5.09 \times 10^{-18} \mathrm{~cm}^{3} \mathrm{~s}^{-1}, \beta=-0.71\right.$, and $\gamma=11.6 \mathrm{~K}$; Brownsword et al. 1997).

In addition to radiative association, we list radiative recombination reactions of $\mathrm{C}^{+}$and $\mathrm{CH}_{3}^{+}$in the same group in Table 3 . The radiative recombination of ionized carbon is a key reaction for the evolution of $\mathrm{C}^{+}$. Its rate coefficient at room temperature is $4.67 \times 10^{-12} \mathrm{~cm}^{3} \mathrm{~s}^{-1}$ with an inverse temperature exponent of 0.6 (Woodall et al. 2007). The assumed uncertainty of this rate (the factor of 10) in osu.2007 is in contrast to the results of Nahar \& Pradhan (1997), who have theoretically predicted this rate value and found that the corresponding uncertainty is only $\sim 10 \%$. In the RATE 06 database the uncertainty of this rate constant is set to $50 \%$.

Finally, the radiative recombination of $\mathrm{CH}_{3}^{+}$is the least probable channel in the reactive collisions between methyl ions and electrons. The rate of this process is estimated to be much higher than for other recombination reactions involving atomic species, particularly in cold regions, and might be accurately determined in laboratory $\left(\alpha=1.1 \times 10^{-10} \mathrm{~cm}^{3} \mathrm{~s}^{-1}, \beta=-0.5\right.$; Millar et al. 1997; Le Teuff et al. 2000; Woodall et al. 2007). In the osu.2007 database, this rate has a steeper dependence on temperature, $\beta=$ -0.7 (Smith et al. 2004). We conclude that RA reactions require a particular attention because their rate uncertainties are often large and not well known so that the modeling results can be heavily affected.

\subsubsection{Cosmic-Ray Ionization}

The next most important reactions that contribute strongly to the resulting abundance scatter of many molecules are the cosmic-ray ionization of molecular hydrogen and helium atoms. The cosmic-ray particles able to penetrate deeply into the disk are mostly high-energy protons $(E \gtrsim 100 \mathrm{MeV})$ and heavy nuclei (e.g., iron, $E \sim 1 \mathrm{GeV}$; Dalgarno \& McCray 1972; Leger et al. 1985; Dolginov \& Stepinski 1994). Direct ionization initiates chemical evolution in dark and cold regions where cosmic-ray particles remain the main ionization source (Herbst \& Klemperer 1973). In the disk this is the outer midplane at $r \gtrsim 30 \mathrm{AU}$ and the lower part of the intermediate layer (Gammie 1996; Semenov et al. 2004). Moreover, since we added the X-ray ionization rate to the CRP rate value in an attempt to crudely treat the effect of high-energy stellar radiation, it is not surprising that high correlation coefficients for some CRP-ionization reactions are reached in the disk atmosphere and intermediate layer at $r \lesssim 100 \mathrm{AU}$ for several key species, such as $\mathrm{CO}$ (see Fig. 4). A more accurate approach to model X-ray chemistry in disks is required (Neufeld et al. 1994; Maloney et al. 1996; Lepp \& Dalgarno 1996; Stäuber et al. 2005).

It is natural that because the cosmic-ray ionization of $\mathrm{H}_{2}$ and $\mathrm{He}$ is the primal chemical process, it is also the key factor for the accumulation of abundance scatters. In the RATE 06 database the corresponding rate coefficients are estimated to be accurate within a factor of 2 (Woodall et al. 2007). However, this rate uncertainty has two origins.

First, there is the physical uncertainty that is related to our limited knowledge of the absolute CRP flux and its penetrating ability, which may vary in different astrophysical objects (Spitzer \& Tomasko 1968; Dolginov \& Stepinski 1994; Caselli et al. 1998). While the direct ionization process driven by CRPs is well studied and the corresponding cross sections and rates are well known (Solomon \& Werner 1971), the second chemical uncertainty resides in the treatment of the ionization by energetic secondary electrons (Glassgold \& Langer 1973). Here the elemental composition of the gas plays a decisive role (Ilgner \& Nelson 2006).

The CRP-induced ionization rate of $\mathrm{H}_{2}$ currently used in all UMIST and OSU databases was taken from the Ph.D. thesis of Black (1975). It was computed for a mixture of atomic and molecular hydrogen, but new calculations for a mixture of hydrogen and helium would be required in order to have a more consistent rate value (see discussion in Wakelam et al. 2006b). Other CRPionization reactions listed in Table 3 do not have a reference in the RATE 06 database so it is hard to judge how accurate their rates are.

Apparently, the rate uncertainty of the CRP-driven reactions can be partially eliminated by more accurate modeling of the full cascade of ionizing events, but the physical uncertainty will still remain, which needs more effort from observers. Wakelam et al. (2006b) have demonstrated how crucial this may be for the results of pure gas-phase chemical models of static molecular clouds.

\subsubsection{Photoreactions}

Another set of chemical processes with problematic rates are photodissociation (PD) and photoionization (PI) reactions, as listed in Table 3. These reactions are important in those regions where UV photons are either able to penetrate (the disk atmosphere and intermediate layer) or are induced by cosmic-ray particles (disk midplane; Prasad \& Tarafdar 1983). High-energy UV photons destroy or ionize gas-phase molecules and allow surface species to desorb back into the gas phase.

While both the PD and PI cross sections for many species can be measured or calculated with a rather good accuracy of $\lesssim 50 \%$ (van Dishoeck 1988; van Dishoeck et al. 2006), the major difficulty in obtaining accurate photorates is our limited knowledge of the UV radiation field inside protoplanetary disks. Bergin et al. (2003) and van Dishoeck et al. (2006) have shown that many $\mathrm{T}$ Tauri stars emit strong, non-blackbody UV radiation. Their spectra resemble that of the interstellar (IS) UV radiation field, with a large fraction of the UV flux emitted in the Ly $\alpha$ line. Van Zadelhoff et al. (2003) have demonstrated the importance of scattering for the UV penetration deep into the disk interior. Furthermore, for the two most abundant molecules, $\mathrm{H}_{2}$ and $\mathrm{CO}$, that effectively dissociate through partly overlapping lines shortward of about $1120 \AA$, the optical thickness in these lines can become so high that self-shielding and mutual shielding have to be taken into account (Dalgarno \& Stephens 1970; van Dishoeck 1988; Lee et al. 1996; Jonkheid et al. 2006).

In the UMIST and OSU databases, the photorates have mostly been adopted from the compilation of van Dishoeck (1988) and Roberge et al. (1991), where they have been computed using a simple plane-parallel approach and the IS UV radiation field of Draine (1978). The IS UV flux is characteristic of a diluted radiation field from an early B star $\left(T_{\text {eff }} \sim 30,000 \mathrm{~K}\right)$ located at a distance of $\sim 10 \mathrm{pc}$, with a cutoff at $912 \AA$ due to absorption by 
interstellar atomic hydrogen (Spitzer 1978; van Dishoeck 1994). Obviously, it is not representative of the UV radiation from much cooler Herbig Ae and T Tauri stars surrounded by circumstellar disks. Recently, van Dishoeck et al. (2006) have recalculated the corresponding PD and PI rates, using more appropriate UV spectral distributions typical of Herbig Ae, T Tauri, and Sun-like stars.

The 10 problematic photodissociation and photoionization reactions found by the sensitivity analysis are involved in either the formation of key species $\left(\mathrm{C}^{+}\right.$and $\left.\mathrm{CN}\right)$, their destruction $(\mathrm{CO}, \mathrm{CN}$, $\mathrm{CS}, \mathrm{NH}_{3}$, and $\mathrm{HCN}$ ), or the destruction of parental molecules $\left(\mathrm{OH}, \mathrm{CH}\right.$, and $\left.\mathrm{CH}_{2}\right)$. Their rates in the RATE 06 database are in general inaccurate by a factor of 2 , with some exceptions. The photodissociation of $\mathrm{CO}$ proceeds indirectly, through predissociation, and depends on the amount of molecular hydrogen and $\mathrm{CO}$ molecules in the line of sight to the source of UV radiation (van Dishoeck 1987), and thus has a rate uncertainty of a factor of 10 (see Table 3). In contrast, the rates of direct photodissociation of $\mathrm{NH}_{3}, \mathrm{OH}$, and $\mathrm{HCN}$ are inaccurate by the factor of 1.5 only (Roberge et al. 1991). Despite such small error bars, the latter three reactions were identified as problematic reactions for disk chemistry.

\subsubsection{Ion-Neutral and Neutral-Neutral Reactions}

Ion-neutral (IN) and neutral-neutral (NN) reactions form the largest fraction of the problematic reactions (32 out of 56) for disk chemistry, but their individual importance is not as significant as for CRI and RA reactions (Table 3). Ion-neutral reactions are usually exothermic and rapid, and lead to the formation of new species by bond rearrangement. Their rate coefficients can often be obtained by simple Langevin theory, which relates the isotropic polarizability of the species and its reduced mass (Clary 1988). For molecules that possess a large dipole moment, the rate values can be significantly enhanced at low temperature by the long-distance Coulomb attraction between the positive ion and negatively charged side of the molecule (Adams et al. 1985; Clary 1985). Moreover, many NN reactions with small barriers or without a barrier can also be fast under interstellar conditions. The rates of most radical-radical reactions and even some radical-stable neutral reactions are controlled by long-range attractive forces and thus do not get smaller at low temperatures (see, e.g., Smith 1988; Sims et al. 1993, 1994b; Canosa et al. 1997). The typical rate coefficient for these reactions is about an order of magnitude lower than for ion-neutral processes. The rates of some of the fast $\mathrm{NN}$ reactions at low temperatures have been measured in the laboratory and predicted theoretically (e.g., Clary et al. 1994; Sims et al. 1994a; Chastaing et al. 2001; Smith et al. 2004).

In RATE 06, most of the IN and NN rate coefficients are uncertain by a factor of 2 and less, up to an accuracy of $25 \%$ (Table 3). However, a major ambiguity that resides in the IN rate constants is the treatment of their temperature dependence. In two versions of the RATE 06 database the rate value for the reaction involving a polar molecule and a molecular ion can have a negative dependence on temperature $(\beta=-0.5$; adopted in this work) or no temperature dependence $(\beta=0)$, while its rate coefficient fits the value at room temperature. In contrast, in the OSU network the corresponding IN rates are based on lowtemperature theoretical estimations that are most accurate at $\sim 10 \mathrm{~K}$ even though the listed reaction rate coefficients are scaled with respect to $300 \mathrm{~K}$ with $\beta \sim-0.5$. Two problematic reactions involving a polar molecule and an ion with significantly different rates in RATE 06 and osu.2007 are those between $\mathrm{C}^{+}$and $\mathrm{NH}$, forming $\mathrm{CN}^{+}$and $\mathrm{H}\left(\alpha_{\text {osu.2007 }}=4.6 \times\right.$ $10^{-9} \mathrm{~cm}^{3} \mathrm{~s}^{-1}$ vs. $\alpha_{\text {RATE0 } 6}=7.8 \times 10^{-10} \mathrm{~cm}^{3} \mathrm{~s}^{-1}$ ) and between
$\mathrm{S}^{+}$and $\mathrm{CH}$, producing $\mathrm{CS}^{+}$and $\mathrm{H}\left(\alpha_{\text {osu. } 2007}=4.4 \times 10^{-9} \mathrm{~cm}^{3} \mathrm{~s}^{-1}\right.$ vs. $\left.\alpha_{\text {RATE } 06}=6.2 \times 10^{-10} \mathrm{~cm}^{3} \mathrm{~s}^{-1}\right)$.

Other IN reactions with different rates in the RATE 06 and osu.2007 databases are reactions involving an atom and a molecular ion. The breakup of molecular hydrogen on collision with helium ion involves a small barrier of $35 \mathrm{~K}$ and $\alpha=3.7 \times$ $10^{-14} \mathrm{~cm}^{3} \mathrm{~s}^{-1}$ in RATE 06 (the relevant temperature range is between 10 and $300 \mathrm{~K}$; Woodall et al. 2007), while in osu.2007 the same rate coefficient is about 5 times smaller and has no barrier (Smith et al. 2004). However, this results in difference of the osu.2007 and RATE 06 rate values of an order of magnitude at most.

Similarly, the OSU reaction between molecular hydrogen and ionized ammonia produces hydrogen atom and protonated ammonia with a rate coefficient of $1.5 \times 10^{-14} \mathrm{~cm}^{3} \mathrm{~s}^{-1}$ and a steep temperature dependence $(\beta=-1.5)$. In RATE 06 this reaction has a non-Langevin rate with $\beta=0$. At temperatures below $20 \mathrm{~K}$ its rate coefficient is twice as large, $\alpha=3.36 \times 10^{-14} \mathrm{~cm}^{3} \mathrm{~s}^{-1}$, and a negative activation barrier of $-35.7 \mathrm{~K}$ exists (Adams \& Smith 1984). At temperatures between 20 and $300 \mathrm{~K}$ this reaction has no barrier and $\alpha=2 \times 10^{-13} \mathrm{~cm}^{3} \mathrm{~s}^{-1}$. For temperatures above $300 \mathrm{~K}$ the rate coefficient is $1.7 \times 10^{-11} \mathrm{~cm}^{3} \mathrm{~s}^{-1}$ and a barrier is about $1000 \mathrm{~K}$ (Fehsenfeld et al. 1975). Consequently, the osu.2007 and RATE 06 rate values can differ by up to one order of magnitude.

Finally, the $\mathrm{IN}$ reaction between $\mathrm{H}_{3}^{+}$and $\mathrm{O}$ produces only $\mathrm{OH}^{+}$ and $\mathrm{H}_{2}$ in OSU, but in RATE 06 a second, less probable alternative channel that leads to the production of ionized water and atomic hydrogen is given (Milligan \& McEwan 2000). Note that the four IN reactions with the smallest rate uncertainty of $25 \%$ are still problematic for disk chemistry. Such high sensitivity of the disk modeling results to the rates of these reactions is caused by their importance for the evolution of $\mathrm{CO}, \mathrm{HCO}^{+}$, and $\mathrm{HCN}$. The most important one is the IN reaction between helium ions and $\mathrm{CO}$, which leads to the hypersensitivity of the final abundances of many carbon-bearing species in the inner part of the disk intermediate layer (see Fig. 2). The other reactions include the primal route to the formation of $\mathrm{HCO}^{+}$and destruction of $\mathrm{CO}: \mathrm{H}_{3}^{+}+\mathrm{CO} \rightarrow \mathrm{HCO}^{+}+\mathrm{H}_{2}\left(\alpha=1.7 \times 10^{-9} \mathrm{~cm}^{3} \mathrm{~s}^{-1} ; \mathrm{Kim}\right.$ et al. 1975), as well as the destruction of molecular nitrogen by ionized helium $\left(\alpha=6.4 \times 10^{-10} \mathrm{~cm}^{3} \mathrm{~s}^{-1}\right.$; Woodall et al. 2007) and destruction of $\mathrm{HCN}$ by ionized carbon $\left(\alpha=3.1 \times 10^{-9} \mathrm{~cm}^{3} \mathrm{~s}^{-1}\right.$, $\beta=-0.5$; Clary et al. 1985).

All of the 15 problematic $\mathrm{NN}$ reactions for disk chemistry are reactions involving atoms $(\mathrm{O}, \mathrm{N}, \mathrm{C}, \mathrm{H}, \mathrm{S})$ and either light hydrocarbons $\left(\mathrm{C}_{n} \mathrm{H}_{m}\right.$, where $n=1$ or 2 and $\left.m=1-3\right)$ or $\mathrm{CN}$, $\mathrm{HCO}$, and $\mathrm{H}_{2} \mathrm{CO}$. In contrast to the IN reactions, the NN reactions are mainly important for the chemical evolution of the key species in warm disk regions, in particular reaction with atomic oxygen (Tscharnuter \& Gail 2007). Although their rates are rather accurate, with uncertainties that are typically not higher than $50 \%$, some of the NN rates are still different in RATE 06 and osu. 2007 databases.

The NN reaction between $\mathrm{N}$ and HCO produces only OCN and $\mathrm{H}$ in osu.2007, with a rate coefficient of $10^{-10} \mathrm{~cm}^{3} \mathrm{~s}^{-1}$ (Smith et al. 2004). In RATE 06 this reaction has in addition two other channels that form either $\mathrm{HCN}$ and $\mathrm{O}\left(\alpha=1.7 \times 10^{-10} \mathrm{~cm}^{3} \mathrm{~s}^{-1}\right)$ or $\mathrm{CO}$ and $\mathrm{NH}\left(\alpha=5.7 \times 10^{-12} \mathrm{~cm}^{3} \mathrm{~s}^{-1}, \beta=0.5\right.$, and $\gamma=$ $1000 \mathrm{~K}$; Woodall et al. 2007).

Another reaction in the list is $\mathrm{H}+\mathrm{CH}$, which leads to $\mathrm{C}$ and $\mathrm{H}_{2}$. In osu.2007, the rate coefficient has a weak dependence on temperature and no barrier $\left(\alpha=2.7 \times 10^{-11} \mathrm{~cm}^{3} \mathrm{~s}^{-1}, \beta=0.38\right.$; Le Teuff et al. 2000), while in RATE 06 this rate has $\beta=0$ and an $80 \mathrm{~K}$ barrier $\left(\alpha=1.3 \times 10^{-10} \mathrm{~cm}^{3} \mathrm{~s}^{-1}\right.$; Woodall et al. 2007). 
Finally, the $\mathrm{NN}$ reaction between $\mathrm{H}$ and $\mathrm{CH}_{2}$, which forms $\mathrm{CH}$ and $\mathrm{H}_{2}$, has the osu.2007 rate coefficient of $2.7 \times 10^{-10} \mathrm{~cm}^{3} \mathrm{~s}^{-1}$ taken from the UMIST 95 database, but this value is only $6.6 \times$ $10^{-11} \mathrm{~cm}^{3} \mathrm{~s}^{-1}$ in RATE 06.

We conclude that the reaction rates of many important IN and $\mathrm{NN}$ reactions are not known accurately enough, as a major controversy in their temperature dependence still persists. This is particularly true for the reactions involving an ion and a polar molecule, whose rates in RATE 06 can differ by a factor of $\sim 5$ at $10 \mathrm{~K}$ for the dipole/non-dipole versions of this ratefile. The ionpolar rates in osu. 2007 can be larger than those in the dipole version of RATE06 by another factor of 4-5, especially for linear neutral reactants, in which allowance is made for subthermal rotational populations via the so-called locked-dipole approach.

\subsubsection{Dissociative Recombination}

Molecular ions are efficiently converted into other, less complex molecules via dissociative recombination (DR) with electrons or charged grains. Similarly to IN reactions, these processes are especially fast at low temperatures with typical rate coefficients of about $10^{-6} \mathrm{~cm}^{-3} \mathrm{~s}^{-1}$ at $10 \mathrm{~K}$ due to the long-range Coulomb attraction. The DR rates can be measured rather accurately with afterglow and storage ring techniques (FlorescuMitchell \& Mitchell 2006). However, the sensitivity of their branching ratios with respect to the temperature is mostly unexplored and may not be described by a simple power law at low $T$ (Petrignani et al. 2005).

A strong influence of the DR rates and branching ratios on the results of chemical models of molecular clouds has been found (Millar et al. 1988; Semaniak et al. 2001; Geppert et al. 2005a). Our sensitivity method picked up two DR reactions that introduce significant uncertainties in computed abundances in protoplanetary disks. These include dissociative recombination of protonated ammonia into ammonia and hydrogen atoms (50th place out of 56), and a main destruction channel for $\mathrm{HCO}^{+}(19$ th place, see Table 3). Note that their rates are supposed to be known within an uncertainty of $25 \%$.

The DR of $\mathrm{HCO}^{+}$in RATE 06 has one dissociative channel to $\mathrm{CO}$ and $\mathrm{H}$ with the total rate coefficient of $2.4 \times 10^{-7} \mathrm{~cm}^{3} \mathrm{~s}^{-1}$ and $\beta=-0.69$ (Mitchell 1990). In the osu.2007 database two additional channels are listed, which lead to the formation of either $\mathrm{OH}$ and $\mathrm{C}$ or $\mathrm{CH}$ and $\mathrm{O}$ but with a much smaller probability of $\sim 4 \%$ each. The DR of $\mathrm{NH}_{4}^{+}$in both RATE 06 and osu.2007 involves three branching channels into $\mathrm{NH}_{2}$ and either two hydrogen atoms $(21 \%)$ or one molecular hydrogen $(10 \%)$, or ammonia and atomic hydrogen $(69 \%)$ with the total rate of $1.5 \times$
$10^{-6} \mathrm{~cm}^{3} \mathrm{~s}^{-1}$ and $\beta=-0.5$ (Vikor et al. 1999). Still, more effort needs to be invested into the (re)investigation of the DR branching ratios and their temperature-dependence, especially at low $\sim 10-20 \mathrm{~K}$ temperatures.

\section{SUMMARY AND CONCLUSIONS}

The influence of gas-phase reaction rate uncertainties on the results of disk chemical modeling has been studied with a Monte Carlo method. The rate coefficients in the RATE 06 network were varied 8000 times within their uncertainty limits using a lognormal distribution. Sets of abundances, column densities, and their error bars were computed for a number of key species in protoplanetary disks. We found that typical uncertainties of the molecular column densities do not exceed a factor of 3-4 even for the largest of the key molecules, which is comparable with observational uncertainties. The column densities of $\mathrm{CO}, \mathrm{C}^{+}$, $\mathrm{H}_{3}^{+}, \mathrm{H}_{2} \mathrm{O}, \mathrm{NH}_{3}, \mathrm{~N}_{2} \mathrm{H}^{+}$, and $\mathrm{HCNH}^{+}$have particularly small error bars. A straightforward correlation analysis between molecular abundances and reaction rates was performed for the entire evolutionary time, and the most problematic reactions involving $\mathrm{CO}, \mathrm{C}^{+}, \mathrm{CS}, \mathrm{HCO}^{+}, \mathrm{H}_{2} \mathrm{CO}, \mathrm{CN}, \mathrm{HCN}$, and $\mathrm{NH}_{3}$ were identified. We showed that the rate coefficients of about a hundred chemical reactions constituting only a few percent of the entire RATE 06 database need to be determined more accurately in order to significantly decrease uncertainties in the modeled abundances and column densities of the key observable molecules. We argue that it is worthwhile to (re)investigate, either experimentally or theoretically, the rate coefficients of basic radiative association and cosmic-ray ionization reactions as well as the temperature dependence of key ion-neutral and neutral-neutral reactions, and the branching ratios and products of dissociative recombination processes. The rate uncertainties of cosmic-ray ionization and photoreactions are partly due to uncertain physical parameters such as the CRP flux and UV penetration, and thus can be decreased with better physical models and observational data.

A.V. appreciates financial support through a grant from the Dynasty Foundation and IMPRS fellowship. E. H. acknowledges the support of the National Science Foundation for his research program in astrochemistry. A. S. acknowledges the support of the Russian Foundation for Basic Research (grant 07-02-00628a). Authors are thankful to the anonymous referee for valuable comments and suggestions. This research has made use of NASA's Astrophysics Data System.
Adams, N. G., \& Smith, D. 1984, Int. J. Mass Spectrom. Ion Processes, 61, 133 Adams, N. G., Smith, D., \& Clary, D. C. 1985, ApJ, 296, L31

Aikawa, Y., \& Herbst, E. 1999, A\&A, 351, 233

Aikawa, Y., Momose, M., Thi, W.-F., van Zadelhoff, G.-J., Qi, C., Blake, G. A., \& van Dishoeck, E. F. 2003, PASJ, 55, 11

Aikawa, Y., \& Nomura, H. 2006, ApJ, 642, 1152

Aikawa, Y., van Zadelhoff, G. J., van Dishoeck, E. F., \& Herbst, E. 2002, A\&A, 386,622

Bates, D. R. 1951, MNRAS, 111, 303

Bates, D. R., \& Herbst, E. 1988, in Proc. ASSL 146, Rate Coefficients in Astrochemistry, ed. T. J. Millar \& D. A. Williams (Dordrecht: Kluwer), 17

Bergin, E., Calvet, N., D’Alessio, P., \& Herczeg, G. J. 2003, ApJ, 591, L159

Bisschop, S. E., Fraser, H. J., Öberg, K. I., van Dishoeck, E. F., \& Schlemmer, S. 2006, A\&A, 449, 1297

Black, J. H. 1975, Ph.D. thesis, Harvard Univ.

Boger, G. I., \& Sternberg, A. 2006, ApJ, 645, 314

Brownsword, R. A., Sims, I. R., Smith, I. W. M., Stewart, D. W. A., Canosa, A., \& Rowe, B. R. 1997, ApJ, 485, 195

\section{EFERENCES}

Canosa, A., Sims, I. R., Travers, D., Smith, I. W. M., \& Rowe, B. R. 1997, A\&A, 323, 644

Caselli, P., Walmsley, C. M., Terzieva, R., \& Herbst, E. 1998, ApJ, 499, 234

Chastaing, D., Le Picard, S. D., Sims, I. R., \& Smith, I. W. M. 2001, A\&A, 365,241

Clary, D. C. 1985, Molec. Phys., 54, 605

1988, in Proc. ASSL 146, Rate Coefficients in Astrochemistry, ed. T. J. Millar \& D. A. Williams (Dordrecht: Kluwer), 1

Clary, D. C., Haider, N., Husain, D., \& Kabir, M. 1994, ApJ, 422, 416

Clary, D. C., Smith, D., \& Adams, N. G. 1985, Chem. Phys. Lett., 119, 320

D’Alessio, P., Calvet, N., Hartmann, L., Lizano, S., \& Cantó, J. 1999, ApJ, 527, 893

Dalgarno, A., \& McCray, R. A. 1972, ARA\&A, 10, 375

Dalgarno, A., \& Stephens, T. L. 1970, ApJ, 160, L107

Dartois, E., Dutrey, A., \& Guilloteau, S. 2003, A\&A, 399, 773

Dobrijevic, M., Ollivier, J. L., Billebaud, F., Brillet, J., \& Parisot, J. P. 2003, A\&A, 398, 335

Dobrijevic, M., \& Parisot, J. P. 1998, Planet. Space Sci., 46, 491

Dolginov, A. Z., \& Stepinski, T. F. 1994, ApJ, 427, 377 
Draine, B. T. 1978, ApJS, 36, 595

Dutrey, A., Guilloteau, S., \& Guelin, M. 1997, A\&A, 317, L55

Dutrey, A., et al. 2007, A\&A, 464, 615

Fehsenfeld, F. C., Lindinger, W., Schmeltekopf, A. L., Albritton, D. L., \& Ferguson, E. E. 1975, J. Chem. Phys., 62, 2001

Finocchi, F., \& Gail, H.-P. 1997, A\&A, 327, 825

Florescu-Mitchell, A. I., \& Mitchell, J. B. A. 2006, Phys. Rep., 430, 277

Gammie, C. F. 1996, ApJ, 457, 355

Garrod, R. T., \& Herbst, E. 2006, A\&A, 457, 927

Geppert, W. D., et al. 2005a, J. Phys. Conf. Ser. 4, 26

Gerlich, D. 1995, Phys. Scr., 1995, 256

Glassgold, A. E., Feigelson, E. D., Montmerle, T., \& Wolk, S. 2005, in ASP Conf. Ser. 341, Chondrites and the Protoplanetary Disk, ed. A. N. Krot, E. R. D. Scott, \& B. Reipurth (San Francisco: ASP), 165

Glassgold, A. E., \& Langer, W. D. 1973, ApJ, 186, 859

Glassgold, A. E., Najita, J., \& Igea, J. 1997a, ApJ, 480, 344 1997b, ApJ, 485, 920

Hasegawa, T. I., \& Herbst, E. 1993, MNRAS, 263, 589

Herbst, E. 1980, ApJ, 241, 197 1985, ApJ, 291, 226

Herbst, E., \& Klemperer, W. 1973, ApJ, 185, 505

Hollenbach, D., \& McKee, C. F. 1979, ApJS, 41, 555

Hollis, J. M., Lovas, F. J., Remijan, A. J., Jewell, P. R., Ilyushin, V. V., \& Kleiner, I. 2006, ApJ, 643, L25

Ilgner, M., Henning, T., Markwick, A. J., \& Millar, T. J. 2004, A\&A, 415, 643 Ilgner, M., \& Nelson, R. P. 2006, A\&A, 445, 205

Ishii, K., Tajima, A., Taketsugu, T., \& Yamashita, K. 2006, ApJ, 636, 927

Izzard, R. G., Lugaro, M., Karakas, A. I., Iliadis, C., \& van Raai, M. 2007, A\&A, 466, 641

Jonkheid, B., Kamp, I., Augereau, J.-C., \& van Dishoeck, E. F. 2006, A\&A, 453,163

Kastner, J. H., Zuckerman, B., Weintraub, D. A., \& Forveille, T. 1997, Science, 277,67

Kim, J. K., Theard, L. P., \& Huntress, W. T., Jr. 1975, Chem. Phys. Lett., 32, 610

Lee, H.-H., Herbst, E., Pineau des Forêts, G., Roueff, E., \& Le Bourlot, J. 1996, A\&A, 311, 690

Lee, H.-H., Roueff, E., Pineau des Forêts, G., Shalabiea, O. M., Terzieva, R., \& Herbst, E. 1998, A\&A, 334, 1047

Leger, A., Jura, M., \& Omont, A. 1985, A\&A, 144, 147

Lepp, S., \& Dalgarno, A. 1996, A\&A, 306, L21

Le Teuff, Y. H., Millar, T. J., \& Markwick, A. J. 2000, A\&AS, 146, 157

Maloney, P. R., Hollenbach, D. J., \& Tielens, A. G. G. M. 1996, ApJ, 466, 561

Markwick, A. J., Ilgner, M., Millar, T. J., \& Henning, T. 2002, A\&A, 385, 632

Millar, T. J., Defrees, D. J., McLean, A. D., \& Herbst, E. 1988, A\&A, 194, 250

Millar, T. J., Farquhar, P. R. A., \& Willacy, K. 1997, A\&AS, 121, 139

Milligan, D. B., \& McEwan, M. J. 2000, Chem. Phys. Lett., 319, 482

Mitchell, J. B. A. 1990, Phys. Rep., 186, 215

Nahar, S. N., \& Pradhan, A. K. 1997, ApJS, 111, 339

Neufeld, D. A., Maloney, P. R., \& Conger, S. 1994, ApJ, 436, L127

Öjekull, J., et al. 2006, J. Chem. Phys., 125, 194306

Petrignani, A., van der Zande, W. J., Cosby, P. C., Hellberg, F., Thomas, R. D., \& Larsson, M. 2005, J. Chem. Phys., 122, 014302

Pety, J., Gueth, F., Guilloteau, S., \& Dutrey, A. 2006, A\&A, 458, 841

Piétu, V., Dutrey, A., \& Guilloteau, S. 2007, A\&A, 467, 163

Pineau des Forêts, G., Roueff, E., \& Flower, D. R. 1992, MNRAS, 258, 45P

Prasad, S. S., \& Huntress, W. T., Jr. 1980, ApJS, 43, 1

Prasad, S. S., \& Tarafdar, S. P. 1983, ApJ, 267, 603

Qi, C., Kessler, J. E., Koerner, D. W., Sargent, A. I., \& Blake, G. A. 2003, ApJ, 597, 986

Qi, C., Wilner, D. J., Blake, G. A., Bourke, T. L., Hogerheijde, M., \& Ho, P. T. P. 2005, in IAU Symp. 235, Astrochemistry: Recent Successes and Current Challenges, ed. D. C. Lis, G. A. Blake, \& E. Herbst (Cambridge: Cambridge Univ. Press), 231
Roberge, W. G., Jones, D., Lepp, S., \& Dalgarno, A. 1991, ApJS, 77, 287 Semaniak, J., et al. 2001, ApJS, 135, 275

Semenov, D., Henning, T., Helling, C., Ilgner, M., \& Sedlmayr, E. 2003, A\&A, 410,611

Semenov, D., Pavlyuchenkov, Y., Schreyer, K., Henning, T., Dullemond, C., \& Bacmann, A. 2005, ApJ, 621, 853

Semenov, D., Wiebe, D., \& Henning, T. 2004, A\&A, 417, 93

Shalabiea, O. M., \& Greenberg, J. M. 1995, A\&A, 296, 779

Simon, M., Dutrey, A., \& Guilloteau, S. 2000, ApJ, 545, 1034

Sims, I. R., Queffelec, J.-L., Defrance, A., Rebrion-Rowe, C., Travers, D., Bocherel, P., Rowe, B. R., \& Smith, I. W. M. 1994a, J. Chem. Phys., 100, 4229

Sims, I. R., Queffelec, J.-L., Travers, D., Rowe, B. R., Herbert, L. B., Karthauser, J., \& Smith, I. W. M. 1993, Chem. Phys. Lett., 211, 461

Sims, I. R., Smith, I. W. M., Clary, D. C., Bocherel, P., \& Rowe, B. R. 1994b, J. Chem. Phys., 101, 1748

Singh, P. D., \& Andreazza, C. M. 2000, ApJ, 537, 261

Smith, I. W. M. 1988, in Proc. ASSL 146, Rate Coefficients in Astrochemistry, ed. T. J. Millar \& D. A. Williams (Dordrecht: Kluwer), 106 1989, ApJ, 347, 282

Smith, I. W. M., Herbst, E., \& Chang, Q. 2004, MNRAS, 350, 323

Snyder, L. E., Buhl, D., Schwartz, P. R., Clark, F. O., Johnson, D. R., Lovas, F. J., \& Giguere, P. T. 1974, ApJ, 191, L79

Solomon, P. M., \& Werner, M. W. 1971, ApJ, 165, 41

Spitzer, L. 1978, Physical Processes in the Interstellar Medium (New York: Wiley)

Spitzer, L. J., \& Tomasko, M. G. 1968, ApJ, 152, 971

Stäuber, P., Doty, S. D., van Dishoeck, E. F., \& Benz, A. O. 2005, A\&A, 440, 949

Thi, W.-F., van Zadelhoff, G.-J., \& van Dishoeck, E. F. 2004, A\&A, 425, 955 Tscharnuter, W. M., \& Gail, H.-P. 2007, A\&A, 463, 369

Turanyi, T. 1997, Reliability Engineering and System Safety, 57, 41

van Dishoeck, E. F. 1987, in IAU Symp. 120, Astrochemistry, ed. M. S. Vardya

\& S. P. Tarafdar (Dordrecht: Kluwer), 51

1988, in Proc. ASSL 146, Rate Coefficients in Astrochemistry, ed. T. J.

Millar \& D. A. Williams (Dordrecht : Kluwer), 49

1994, in ASP Conf. Ser. 58, The First Symposium on the Infrared

Cirrus and Diffuse Interstellar Clouds, ed. R. Cutri \& W. Latter (San Francisco: ASP), 319

van Dishoeck, E. F., \& Blake, G. A. 1998, ARA\&A, 36, 317

van Dishoeck, E. F., Jonkheid, B., \& van Hemert, M. C. 2006, in Faraday Discussions 133, Chemical Evolution of the Universe, ed. I. R. Sims \& D. A. Williams (Cambridge: R. Soc. Chem.), 231

van Zadelhoff, G.-J., Aikawa, Y., Hogerheijde, M. R., \& van Dishoeck, E. F. 2003, A\&A, 397, 789

van Zadelhoff, G.-J., van Dishoeck, E. F., Thi, W.-F., \& Blake, G. A. 2001, A\&A, 377, 566

Vasyunin, A. I., Sobolev, A. M., Wiebe, D. S., \& Semenov, D. A. 2004, Astron. Lett., 30, 566 (Paper I)

Vikor, L., et al. 1999, A\&A, 344, 1027

Wakelam, V., Herbst, E., \& Selsis, F. 2006a, A\&A, 451, 551

Wakelam, V., Herbst, E., Selsis, F., \& Massacrier, G. 2006b, A\&A, 459, 813

Wakelam, V., Selsis, F., Herbst, E., \& Caselli, P. 2005, A\&A, 444, 883 (Paper II)

Wiebe, D., Semenov, D., \& Henning, T. 2003, A\&A, 399, 197

Willacy, K., Klahr, H. H., Millar, T. J., \& Henning, T. 1998, A\&A, 338, 995

Willacy, K., \& Langer, W. D. 2000, ApJ, 544, 903

Willacy, K., Langer, W., Allen, M., \& Bryden, G. 2006, ApJ, 644, 1202

Williams, D. A. 1972, Astrophys. Lett., 10, 17

Woodall, J., Agundez, M., Markwick-Kemper, A. J., \& Millar, T. J. 2007, A\&A, 466, 1197 